\title{
CONTRIBUTIONS TO THE CLASSIFICATION OF SIMPLE MODULAR LIE ALGEBRAS
}

\author{
GEORGIA BENKART, J. MARSHALL OSBORN AND HELMUT STRADE
}

\begin{abstract}
We develop results directed towards the problem of classifying the finite-dimensional simple Lie algebras over an algebraically closed field of characteristic $p>7$. A 1-section of such a Lie algebra relative to a torus $T$ of maximal absolute toral rank possesses a unique subalgebra maximal with respect to having a composition series with factors which are abelian or classical simple. In this paper we show that the sum $Q$ of those compositionally classical subalgebras is a subalgebra. This extends to the general case a crucial step in the classification by Block and Wilson of the restricted simple Lie algebras. We derive properties of the filtration which can be constructed using $Q$ and obtain structural information about the 1-sections and 2-sections of $Q$ relative to $T$. We further classify all those algebras in which $Q$ is solvable.
\end{abstract}

\section{INTRODUCTION}

Work on the problem of classifying the finite-dimensional simple Lie algebras over an algebraically closed field of prime characteristic dates from the pioneering studies of Jacobson, Witt, and Zassenhaus in the 1930s. It continues today, and in fact there has been substantial progress on this question in the last few years. It has been known since the 1930s that examples of simple Lie algebras exist in prime characteristic in addition to the classical Lie algebras, which are the analogues of the characteristic zero simple Lie algebras. In [KS1, KS2] Kostrikin and Šafarevič presented a construction which gave families of nonclassical simple Lie algebras. The Lie algebras were described as truncated versions of the infinite-dimensional complex Lie algebras used by Cartan in his study of Lie pseudogroups, and so were termed Lie algebras of Cartan type. Kostrikin and Šafarevič [KS1] conjectured that all restricted simple Lie algebras were classical or Cartan type. In [Kac3] and [W1], Kac and Wilson independently generalized the Kostrikin-Šafarevič construction showing that further examples could be obtained from the Cartan type algebras by changing the differential form or equivalently, by using an automorphism. If one refers to these algebras as Lie algebras of Cartan type also, then one has the generalized Kostrikin-Šafarevič conjecture-every simple Lie algebra over an algebraically closed field of characteristic $p>5$ is classical or Cartan type. There is much evidence to support this conjecture: [MS, Kap, Kac2, Kac3, W1,

Received by the editors June 4, 1990 and, in revised form, October 1, 1991.

1991 Mathematics Subject Classification. Primary 17B50, 17B20.

The first and second authors were supported in part by National Science Foundation Grant \# DMS-8800691. 
W2, W3, BW1, BO]. A major breakthrough came with the result which was announced in 1984 [BW2, BW3, W4] and which appeared in 1988 [BW4] that a simple restricted Lie algebra is classical or Cartan type if $p>7$.

The main tool used to identify a simple Lie algebra is the "Recognition Theorem" (see [Kac1, Kac2, Kac3, W1, and BGP]), which states that if $L$ is a simple Lie algebra having a suitable maximal subalgebra $L_{(0)}$, then $L$ is classical or Cartan type. Recently, Benkart and Gregory [BG] improved this theorem by showing that one of the hypotheses on $L_{(0)}$ (a certain restrictedness assumption) can be dropped.

In establishing their result Block and Wilson proved the following main steps:

(i) There is a maximal torus $T$ relative to which each root $\alpha$ is "proper."

(ii) If $L^{(\alpha)}$ is a 1-section relative to $T$, then one of the following holds:

(a) $L^{(\alpha)} / \operatorname{rad} L^{(\alpha)}=(0)$,

(b) $L^{(\alpha)} / \operatorname{rad} L^{(\alpha)} \cong s l(2)$

(c) $L^{(\alpha)} / \operatorname{rad} L^{(\alpha)} \cong W(1: \underline{\underline{1}})$

(d) $H(2: \underline{\underline{1}})^{(2)} \subseteq L^{(\alpha)} / \operatorname{rad} \bar{L}^{(\alpha)} \subseteq H(2: \underline{\underline{1}})$.

(iii) If $L^{(\alpha, \beta)}$ is a 2 -section relative to $T$, then there is a maximal solvable ideal $I^{(\alpha, \beta)}$ of $L^{(\alpha, \beta)}$ such that $L^{(\alpha, \beta)} / I^{(\alpha, \beta)}$ belongs to a certain list.

(iv) Each 1-section $L^{(\alpha)}$ contains a "maximum compositionally classical" subalgebra $U^{(\alpha)}$.

(v) $Q=\sum_{\alpha} U^{(\alpha)}$ is a subalgebra.

(vi) If $Q=L$, then $L$ is classical.

(vii) If $Q \neq L$, then $Q$ satisfies the hypotheses of the Recognition Theorem; consequently, $L$ is classical or Cartan type.

In the general case a turning point came with Strade's realization that the basic program outlined in (i)-(vii) works for $p>7$ if appropriately modified. Indeed, in [S1] Strade introduces the notion of a torus $T$ of maximal absolute toral rank and establishes that the 1-sections relative to $T$ are as described in (ii). Such a torus may be found having all roots proper [S2]; moreover, if $T$ is a torus of maximal absolute toral rank having all roots proper, then the 2sections may be determined as in (iii) ([S2]; compare Theorem 1.15). Strade further shows in that paper, that (iv) holds in the general case (see Proposition 1.11).

It is the goal of this paper to establish (v) and to determine the Lie algebras in which the subalgebra $Q$ is solvable.

This is not the end to the classification quest, as steps (vi) and (vii) remain to be done in the general case. The remainder of the problem seems to divide naturally into four cases:

(A) $Q=L$ and all 1-sections are solvable.

(B) $Q=L$ and at least one classical 1-section is present,

(C) $Q \neq L$ and no 2-section is of type $H(2: \underline{\underline{1}}: \phi(\tau))^{(1)}$.

(D) $Q \neq L$ and $L$ has a 2 -section of type $H(2: \underline{\underline{1}}: \phi(\tau))^{(1)}$.

In case (B), it has been established by Benkart in [B2] that $L$ is classical if $L$ does not contain any 2-sections of type (6) in Strade's list of 2-sections (Theorem 1.15). In (C), $L$ has been shown to be classical or Cartan type if no 2-sections of type (6) with $S=W(1: \underline{\underline{1}})$ or $H\left(2: \underline{1}^{(2)}\right.$ or type (7) with $S=W(1: \underline{\underline{2}})$ or $H(2:(2,1))^{(2)}$ occur (see [B3]). Since this present paper 
was submitted, Strade [S4-S8] and Strade and Wilson [SW2] have shown that the generalized Kostrikin-Šafarevič conjecture holds in cases (A)-(D). Thus, as announced in [SW1], the generalized Kostrikin-Šafarevič conjecture is true for all $p>7$. The proofs depend on the results of this paper.

Our paper is organized as follows: In $\S 1$ we establish notation, give relevant definitions, and quote needed results. Section 2 investigates how the 1 -sections fit together in the 2 -sections. In $\S 3$ we establish that $Q$ is a subalgebra and derive properties of the filtration which can be constructed using $Q$. The subalgebra $Q$ is $T$-invariant. In $\S 4$ we determine the 1-sections and 2-sections of $Q$ with respect to $T$ and prove that $L$ is isomorphic to $W(1: \underline{\underline{n}})$ if and only if $Q$ is solvable.

\section{NOTATION, BACKGROUND, PRELIMINARY RESULTS}

We consider finite-dimensional Lie algebras $L$ over an algebraically closed field $F$ of characteristic $p>7$. For definitions and facts stated without proof, we refer to [SF, S1, or S2]. Let $K$ be a subalgebra in $L$, and let $\left(L_{p},[p], l\right)$ be any $p$-envelope of $L$. Let $K_{p}$ denote the restricted subalgebra of $L_{p}$ generated by $l(K)$. Then

$$
\operatorname{TR}(K, L):=\max \left\{\operatorname{dim} T \mid T \text { is a torus of } K_{p}+C\left(L_{p}\right) / C\left(L_{p}\right)\right\}
$$

is the (absolute) toral rank of $K$ in $L$. According to [S1], this definition is independent of the $p$-envelope chosen. When $K=L$, we abbreviate the notation $\operatorname{TR}(L, L)$ to $\operatorname{TR}(L)$. A torus with $\operatorname{dim} T=\operatorname{TR}(L)$ is called a torus of maximal absolute toral rank.

Relative to any torus $T \subset L_{p}$ we have the root space decomposition

$$
L=\sum_{\alpha \in \Omega(T)} L_{\alpha}(T)
$$

where $L_{0}(T)=C_{L}(T)$ is the centralizer in $L$ of $T$. If $\alpha_{1}, \ldots, \alpha_{k}$ are $\mathbb{Z}_{p^{-}}$ independent roots, then they determine the $k$-section

$$
L^{\left(\alpha_{1}, \ldots, \alpha_{k}\right)}(T)=\sum_{i_{j} \in \mathbb{Z}_{p}} L_{i_{1} \alpha_{1}+\cdots+i_{k} \alpha_{k}}(T) .
$$

When there is no ambiguity about what torus is being used, we drop "( $T)$ " from the notation for root spaces and sections.

Definition 1.1. A torus $T \subset L_{p}$ is said to be standard with respect to $L$ if $C_{L}(T)=L_{0}(T)$ acts triangulably on $L$.

Remarks. (1) If $T$ is a torus of maximal absolute toral rank in $L_{p}$, and if $T_{0}$ is the unique maximal torus in the center $C\left(L_{p}\right)$ of $L_{p}$, then by Lemma 1.1 of [S2], $T^{\prime}=T+T_{0}$ is the unique maximal torus of $L_{p}$ which contains $T$, and $T^{\prime}$ is a torus of maximal dimension in $L_{p}$. Thus, by replacing $T$ by $T^{\prime}$ if necessary, we see that there is no harm in assuming in what follows that a torus of maximal absolute toral rank is also of maximal dimension in $L_{p}$, and it contains the unique maximal torus of $C\left(L_{p}\right)$. We make this assumption throughout this paper.

(2) Roots relative to any maximal torus $T$ in $L_{p}$ can be extended to $C_{L}(T)$ in the following way. For each $h \in H=C_{L}(T), h^{[p]^{r}}$ is semisimple for 
some value of $r$, and $T+F h^{[p]^{r}}$ is a torus. Therefore, by the maximality of $T, h^{[p]^{r}} \in T$. If $\alpha$ is a root relative to $T$, then $\alpha$ extends to $H$ by letting $\alpha(h)$ be such that $\alpha(h)^{p^{r}}=\alpha\left(h^{[p]^{r}}\right)$. If $T$ is standard, then $H^{(1)}$ acts nilpotently on $L$. Thus, the extension of $\alpha$ to $H$ is linear whenever $T$ is standard.

(3) An algebra $L$ is nilpotent if and only if $\operatorname{TR}(L)=0$. Indeed, if $\operatorname{TR}(L)=$ 0 , then any torus in $L_{p}$ is contained in $C\left(L_{p}\right)$. For each $x \in L$, some $[p]$-power $t:=x^{[p]^{r}}$ is semisimple, and so $t \in C\left(L_{p}\right)$. But then $(\operatorname{ad} x)^{p^{r}}=$ $\operatorname{ad} x^{[p]^{r}}=0$, and Engel's theorem implies that $L$ is nilpotent. Conversely, if $L$ is nilpotent, so is any $p$-envelope $L_{p}$. Any torus in $L_{p}$ is central. Therefore, $\operatorname{TR}(L)=0$.

The following general result will be applied to the study of 1-sections:

Proposition 1.2 [S2, Theorem 1.5]. Let $L=\bigoplus \sum_{i \in G} L_{i}$ be a cyclically graded Lie algebra so that $G$ is a cyclic group and $\left[L_{i}, L_{j}\right] \subseteq L_{i+j}$ for all $i, j \in G$. If $L_{0}$ acts nilpotently on $L$, then $L$ is solvable.

Lemma 1.3. Let $L$ be a Lie algebra, and $T$ be a maximal torus in some $p$ envelope $\left(L_{p},[p], l\right)$. If $L^{(\alpha)}$ is a nonsolvable 1 -section relative to $T$, then $C_{L}(T)$ is a Cartan subalgebra of $L^{(\alpha)}$.

Proof. As the centralizer of a maximal torus, $C_{L_{p}}(T)$ must be a Cartan subalgebra of $L_{p}$. (See, for example, [SF, Chapter 2, Theorem 4.1].) Thus, $C_{L}(T) \subseteq C_{L_{p}}(T)$ implies that $C_{L}(T)$ is nilpotent. Since $L^{(\alpha)}$ is assumed to be nonsolvable, $C_{L}(T)$ must act nonnilpotently on $L^{(\alpha)}$ by Proposition 1.2. Hence, there exists an $h \in C_{L}(T)$ with $j \alpha(h) \neq 0$ for all $j \neq 0$. It follows that the normalizer of $C_{L}(T)$ is just $C_{L}(T)$ itself, and $C_{L}(T)$ is a Cartan subalgebra.

Lemma 1.4. Let $L$ be a Lie algebra, and $T$ be a maximal torus in some $p$ envelope $\left(L_{p},[p], \imath\right)$. Assume that $K=L^{(\alpha)}$ is a 1-section relative to $T$. Then $\operatorname{rad} K$ is $T$-invariant.

Proof. If $K$ is solvable, then clearly $\operatorname{rad} K=K$ is $T$-invariant, so we may assume that $K$ is nonsolvable. Then $C_{L}(T)$ does not act nilpotently on $K$ by Proposition 1.2. Hence there exists an $h \in C_{L}(T)$ such that $\left.\operatorname{ad} h\right|_{K}$ is not nilpotent. Let $t=h^{[p]^{r}} \in C_{L_{p}}(T)$ be the semisimple part of $h$. The maximality of $T$ implies that $t \in T$. Moreover, since $t$ does not centralize $K, T=F t \oplus \operatorname{ker} \alpha$. But $\operatorname{ker} \alpha$ centralizes $K$, and $\operatorname{rad} K$ is invariant under ad $t=(\operatorname{ad} h)^{p^{r}}$. Thus $\operatorname{rad} K$ is $T$-invariant.

Lemma 1.4 was shown to hold when $L$ is simple and $T$ is a torus of maximal absolute toral rank [S2, Theorem 1.8]. The proof given in [S2] invoked the following result instead of Proposition 1.2:

Theorem 1.5 [S2, Theorem 1.4]. Let $L$ be a simple Lie algebra over an algebraically closed field $F$ of characteristic $p>7$ and $T$ be a torus in some p-envelope.

(i) If $C_{L}(T)$ is a nilpotent algebra, then $T$ is standard with respect to $L$.

(ii) Let $T$ be a torus of maximal absolute toral rank and $\alpha \neq 0$ a root with respect to $T$. If $\alpha\left(C_{L}(T)\right)=0$ (i.e., if $C_{L}(T)$ acts nilpotently on $L_{\alpha}$ ), then $L^{(\alpha)}$ is nilpotent and acts triangulably on $L$.

Whenever $T$ is a maximal torus of some $p$-envelope, then $C_{L}(T)$ is a nilpotent algebra. (See the first two lines of the proof of Lemma 1.3 or use Propo- 
sition 3.2 of [S1].) Therefore, as an immediate consequence of Theorem 1.5(i) we have part (a) of the following theorem. Part (b) is Theorem 3.1 of [S2].

Theorem 1.6. Let $L$ be a simple Lie algebra over an algebraically closed field $F$ of characteristic $p>7$, and let $\left(L_{p},[p], l\right)$ be a p-envelope of $L$.

(a) If $T$ is a maximal torus of $L_{p}$, then $T$ is standard with respect to $L$.

(b) If $T$ is a torus of maximal absolute toral rank in $L_{p}$, then $T$ is standard with respect to $L$ and $L_{p}$.

Proposition 1.7 [S1, Theorem 2.6]. Let $L$ be a Lie algebra and $\left(L_{p},[p], l\right)$ a p-envelope of $L$. Suppose $T \subseteq L_{p}$ is a torus of maximal absolute toral rank, and $K$ is a $k$-section with respect to $T$. Then $\operatorname{TR}(K) \leq k$.

Lie algebras $K$ with $\operatorname{TR}(K) \leq 1$ (in particular 1-sections relative to a torus of maximal absolute toral rank) have been described by Strade:

Theorem 1.8 [S2, Theorem 1.7]. Let $K$ be a subalgebra of $L$.

(1) $\operatorname{TR}(K, L)=0$ if and only if $K$ acts nilpotently on $L$.

(2) If $\operatorname{TR}(K)=1$, and if some Cartan subalgebra of $K$ acts triangulably on $K$, then one of the following occurs:

(a) $K / \operatorname{rad} K=(0)$, and $K^{(1)}$ is nilpotent.

(b) $K / \operatorname{rad} K \cong \operatorname{sl}(2)$ and $\operatorname{rad} K$ is nilpotent.

(c) $K / \operatorname{rad} K \cong W(1: \underline{\underline{1}})$ and $\operatorname{rad} K$ is nilpotent.

(d) $H(2: \underline{\underline{1}})^{(2)} \subseteq K / \operatorname{rad} K \subseteq H(2: \underline{\underline{1}})$ and $\operatorname{rad} K$ is nilpotent.

Corollary 1.9. Let $L$ be a Lie algebra, and $\left(L_{p},[p], \imath\right)$ a p-envelope of $L$. Let $T \subset L_{p}$ be a torus of maximal absolute toral rank such that $T$ is standard with respect to $L$, and assume that $K=L^{(\alpha)}$ is a 1-section of $L$ relative to $T$. Then $\operatorname{TR}(K) \leq 1$. Moreover,

(i) if $\operatorname{TR}(K)=0$, then $K$ is nilpotent;

(ii) if $\operatorname{TR}(K)=1$, then one of the following occurs:

(a) $K / \operatorname{rad} K=(0)$.

(b) $K / \operatorname{rad} K \cong \operatorname{sl}(2)$, and $\operatorname{rad} K$ is nilpotent.

(c) $K / \operatorname{rad} K \cong W(1: \underline{\underline{1}})$, and $\operatorname{rad} K$ is nilpotent.

(d) $H(2: \underline{\underline{1}})^{(2)} \subseteq K / \operatorname{rad} K \subseteq H(2: \underline{\underline{1}})$ and $\operatorname{rad} K$ is nilpotent.

Proof. By Proposition 1.7, $\operatorname{TR}(K) \leq 1$. Remark (3) at the beginning of this section gives (i). To obtain the second part, use Lemma 1.3 to see that $C_{L}(T)$ is a Cartan subalgebra of $K$ whenever $K$ is nonsolvable. Because $T$ is assumed to be standard, $C_{L}(T)$ acts triangulably on $K$. The rest follows from Theorem 1.8 .

Definition 1.10. For each root $\alpha$, let $L^{[\alpha]}=L^{[\alpha]}(T):=L^{(\alpha)} / \operatorname{rad} L^{(\alpha)}$. Then $\alpha$ or $L^{(\alpha)}$ is said to be solvable, classical, Witt, or Hamiltonian if $L^{[\alpha]}=$ $(0), L^{[\alpha]} \cong \operatorname{sl}(2), L^{[\alpha]} \cong W(1: \underline{\underline{1}})$, or $H(2: \underline{\underline{1}})^{(2)} \subseteq L^{[\alpha]} \subseteq H(2: \underline{1})$, respectively.

Proposition 1.11 [S2, Proposition 1.9]. Let $K$ be a Lie algebra with a triangulable Cartan subalgebra, and assume $\operatorname{TR}(K) \leq 1$. Then $K$ contains a unique (not necessarily proper) subalgebra $U=U(K) \supseteq \operatorname{rad} K$ of maximal dimension having codimension $\leq 2$, such that $U / \operatorname{rad} U$ is (0) or isomorphic to $s l(2)$. More explicitly, if $\pi: K \rightarrow K / \operatorname{rad} K$ is the canonical projection, then

(i) $U=K$ if $K$ is solvable, or if $K / \operatorname{rad} K \cong s l(2)$; 
(ii) $U=\pi^{-1}\left(\sum_{i>0} F x^{i} d / d x\right)$ if $K / \operatorname{rad} K \cong W(1: \underline{\underline{1}})$;

(iii) $U=\pi^{-1}\left(\left(\sum_{k+l>0} F x^{k \epsilon_{1}+l \epsilon_{2}} D_{1}+\sum_{k+l>0} F x^{k \epsilon_{1}+l \epsilon_{2}} D_{2}\right) \cap(K / \operatorname{rad} K)\right)$ if $H(2: \underline{\underline{1}})^{(2)} \subseteq K / \operatorname{rad} K \subseteq H(2: \underline{\underline{1}})$.

Suppose now that $L$ is a Lie algebra, and $\left(L_{p},[p], l\right)$ a $p$-envelope of $L$. Let $T \subset L_{p}$ be a torus of maximal absolute toral rank such that $T$ is standard with respect to $L$, and assume that $L^{(\alpha)}$ is a 1-section of $L$ relative to $T$. If $\alpha$ is solvable, then we set $U\left(L^{(\alpha)}\right) \stackrel{\text { def }}{=} L^{(\alpha)}$. When $\alpha$ is classical, Witt or Hamiltonian, then $C_{L}(T)$ is a triangulable Cartan subalgebra of $L^{(\alpha)}$ by Lemma 1.3, and Proposition 1.11 applies to give the subalgebra $U\left(L^{(\alpha)}\right)$. We abbreviate the notation $U\left(L^{(\alpha)}\right)$ to $U^{(\alpha)}$ or to $U^{(\alpha)}(T)$ if the torus needs to be distinguished. The subalgebra $U^{(\alpha)}$ (or $U(K)$ in the case of Proposition 1.11) is called the maximum compositionally classical subalgebra of $L^{(\alpha)}$ (of $K$ ). The phrase "compositionally classical" refers to the fact that every composition factor is abelian or classical simple. We consider the following subspace of $L$ spanned by the maximum compositionally classical subalgebras of all the 1 -sections:

$$
Q(L, T):=\sum_{\alpha \in \Omega(T)} U^{(\alpha)}(T)
$$

It is the goal of $\S 3$ to show that $Q=Q(L, T)$ is a subalgebra whenever the torus $T$ is optimal.

Recall that a root $\alpha \neq 0$ (with respect to $T$ ) is termed proper if there is some $j \neq 0$ such that $\alpha\left(\left[L_{j \alpha}(T), L_{-j \alpha}(T)\right]\right)=0$. A torus $T$ is said to be optimal if it has maximal absolute toral rank in $L_{p}$, and if among all tori of maximal absolute toral rank, the number of proper roots with respect to $T$ is maximal. By definition optimal tori necessarily exist. Moreover, according to [S2, Theorem 5.3], if $L$ is a simple Lie algebra over an algebraically closed field of characteristic $p>7$, the roots relative to an optimal torus are all proper. If $T_{0}$ is the unique maximal torus of $C\left(L_{p}\right)$ as above, then $T^{\prime}=T+T_{0}$ is optimal whenever $T$ is. Hence, we may assume in what follows that any optimal torus $T$ is of maximal dimension.

In establishing that $Q$ is a subalgebra we make use of the next theorem concerning optimal tori. This result was proved in [S2, Theorem 7.1], under the hypothesis that $L$ is simple. We replace that assumption with the requirement that $T$ be standard with respect to $L$.

Theorem 1.13 [S2, Theorem 7.1]. Let $L$ be a Lie algebra over an algebraically closed field $F$ of characteristic $p>7$. Let $T$ be an optimal torus of some p-envelope $\left(L_{p},[p], l\right)$ of $L$ which is standard with respect to $L$. Then the maximum compositionally classical subalgebra of every 1-section with respect to $T$ is $T$-invariant.

Proof. We want to show that $U^{(\alpha)}$ is $T$-invariant for each root $\alpha$. If $\alpha$ is solvable, or classical the result is clear, so we may assume that $\alpha$ is Witt or Hamiltonian. Then $H:=C_{L}(T)$ is a Cartan subalgebra of $L^{(\alpha)}$, and there exists an $h \in H$ with $j \alpha(h) \neq 0$ for each $j \neq 0$. If $\pi: L^{(\alpha)} \rightarrow L^{[\alpha]}$ denotes the canonical projection, then $\pi(H)$ is a Cartan subalgebra of $L^{[\alpha]}$. Since roots relative to $\pi(H)$ must be proper, we have in the Witt case that $\pi(H) \subset$ $W(1: \underline{\underline{1}})_{(0)}=\sum_{i \neq 0} x^{i} d / d x$ (see [BW4, Lemma 5.6.3]). But then $U^{(\alpha)}=$ 
$\pi^{-1}\left(W(1: \underline{\underline{1}})_{(0)}\right)$ implies that $H \subset U^{(\alpha)}$. If $x \in U^{(\alpha)}$ and $x=\sum_{j \in \mathbb{Z}_{p}} x_{j \alpha}$, the components, $x_{j \alpha} \in L_{j \alpha}(T)$, must lie in $U^{(\alpha)}$ also. Consequently, $U^{(\alpha)}$ is $T$-invariant.

When $\alpha$ is Hamiltonian, let $S:=H(2: \underline{\underline{1}})^{(2)}$ and $\widehat{H}=\pi(H) \cap S$. Observe that $\left(L^{[\alpha]}\right)^{(2)}=S$, so that $S$ is $T$-invariant, hence, cyclically graded. Since $S$ is simple, $\widehat{H}$ acts nonnilpotently on $S$ by Proposition 1.2, and so is a Cartan subalgebra of $S$. It follows from the properness of the roots (see [BW4, Theorem 1.3.1]) that $\widehat{H} \subset S_{(0)}$, the unique subalgebra of $S$ of codimension 2. As in the Witt case, this implies that $U^{(\alpha)}$ is $T$-invariant.

Corollary 1.14 (Compare [BW4, Lemma 12.1.3]). Let the assumptions be as in Theorem 1.13.

(a) If $\alpha$ is a Witt root, then there is some $i \in \mathbb{Z}_{p}^{*}$ such that $\operatorname{dim} L_{j \alpha} /\left(U^{(\alpha)}\right)_{j \alpha}=$ $\delta_{i, j}$

(b) If $\alpha$ is Hamiltonian, then there is some $i \in \mathbb{Z}_{p}^{*}$ such that $\operatorname{dim} L_{j \alpha} /\left(U^{(\alpha)}\right)_{j \alpha}$ $=\delta_{i, j}+\delta_{-i, j}$.

Consequently, $C_{L}(T) \subset U^{(\alpha)}$ for each root $\alpha$.

Proof. In the Witt case, we have established in the proof of Theorem 1.13 that $H \subset U^{(\alpha)}=\pi^{-1}\left(W(1: \underline{1})_{(0)}\right)$, so that the result follows. In the Hamiltonian case, the definition of $U^{(\alpha)}$ implies the existence of root vectors $u \in L_{i \alpha}, v \in$ $L_{j \alpha}$ such that $L^{(\alpha)}=U^{(\alpha)} \oplus F u \oplus F v$, and $\pi\left(U^{(\alpha)}\right) \subset\left(\sum_{k+l>0} F x^{k \epsilon_{1}+l \epsilon_{2}} D_{1}+\right.$ $\left.\sum_{k+l>0} F x^{k \epsilon_{1}+l \epsilon_{2}} D_{2}\right)$ by Proposition 1.11. Then $T$ acts on $L^{(\alpha)} / \operatorname{rad} L^{(\alpha)}$ as the torus $R:=T /\left\{t \in T \mid\left[t, L^{(\alpha)}\right] \subset \operatorname{rad} L^{(\alpha)}\right\}$, and since roots are proper, $R$ is conjugate to $F\left(x_{1} D_{1}-x_{2} D_{2}\right)$. It follows that $j=-i \neq 0$.

Having obtained information about 1-sections, we turn our attention now to 2-sections. In the general case there are eight possible types of 2-sections:

Theorem 1.15 [S3, Theorem II.2]. Let $L$ be a simple Lie algebra over an algebraically closed field $F$ of characteristic $p>7$. Let $T$ be an optimal torus in some p-envelope $\left(L_{p},[p], l\right)$ of $L$. Let $L^{(\alpha, \beta)}$ denote a 2 -section relative to $T$, and let $I^{(\alpha, \beta)}=I^{(\alpha, \beta)}(T)$ be the maximal solvable ideal in $L^{(\alpha, \beta)}+T$. Assume that $\pi=\pi_{\alpha, \beta}$ is the canonical projection $L^{(\alpha, \beta)}+T \rightarrow\left(L^{(\alpha, \beta)}+T\right) / I^{(\alpha, \beta)}$. Let $K:=K^{(\alpha, \beta)}=\pi\left(L^{(\alpha, \beta)}\right)$ and $\bar{T}=\pi(T)$. Then one of the following cases occurs:

(1) $K=(0)$.

(2) $S \subseteq K+\bar{T} \subseteq(\operatorname{Der} S)^{(1)}$, where $S \in\left\{\operatorname{sl}(2), W(1: \underline{\underline{1}}), H(2: \underline{\underline{1}})^{(2)}\right\}$, and there exists some $\lambda \in \mathbb{Z}_{p} \alpha+\mathbb{Z}_{p} \beta$ such that $K=\pi\left(L^{(\lambda)}\right)$.

(3) $S_{1} \oplus S_{2} \subseteq K+\bar{T} \subseteq\left(\operatorname{Der} S_{1}\right)^{(1)} \oplus\left(\operatorname{Der} S_{2}\right)^{(1)}$ where $S_{1}, S_{2} \in\{\operatorname{sl}(2)$, $W(1: \underline{\underline{1}}), H\left(2: \underline{\underline{1}}^{(2)}\right\}$.

(4) $H\left(2: \underline{\underline{1}}^{(2)} \subseteq K+\bar{T} \subseteq \operatorname{Der} H\left(2: \underline{\underline{1}}^{(2)}\right.\right.$, and $\bar{T} \not \subset H(2: \underline{\underline{1}})$.

(5) $S \otimes A(n: \underline{\underline{1}}) \subseteq K+\bar{T} \subseteq \operatorname{Der}(S \otimes A(n: \underline{\underline{1}}))$, where $S \in\{$ sl(2), $W(1: \underline{1}), H\left(2: \underline{\underline{1}}^{(2)}\right\}, \quad n=1,2$, and $\lambda\left(C_{L}(T)\right) \neq 0$ for all $\lambda \in \mathbb{Z}_{p} \alpha+$ $\mathbb{Z}_{p} \beta-\{0\}$.

(6) $S \otimes A(1: \underline{1}) \subseteq K+\bar{T} \subseteq \operatorname{Der}(S \otimes A(1: \underline{\underline{1}}))$ where $S \in\{s l(2)$, $\left.W(1: \underline{\underline{1}}), H(2: \underline{\underline{1}})^{(\overline{2})}\right\} ;$ and $(S \otimes A(1: \underline{\underline{1}})) \cap \operatorname{rad} K=\bar{S} \otimes x A(1: \underline{\underline{1}})$. 
(7) $S \subseteq K+\bar{T} \subseteq \operatorname{Der} S$, where $S \in\left\{W(1: \underline{\underline{2}}), H(2:(2,1))^{(2)}\right.$, $\left.H(2: \underline{\underline{1}}: \phi(\tau))^{(1)}, \quad H(2: \underline{\underline{1}}: \Delta)\right\}$.

(8) $K=S+C_{K}(\bar{T})$ where $S \in\left\{A_{2}, C_{2}, G_{2}, W(2: \underline{1}), S\left(3: \underline{\underline{1}}^{(1)}\right.\right.$, $\left.H(4: \underline{\underline{1}})^{(2)}, K(3: \underline{\underline{1}})\right\}$.

Explicit information concerning the torus $\bar{T}$ in Theorem 1.15 is derived in [S2, Theorem 7.2] (see also the remarks in Chapter II of [S3]). In the statement of the theorem given in [S2], automorphisms have been suppressed. They are included in the statement of the next theorem, which elaborates on the result of [S2].

Theorem 1.16 [S2, Theorem 7.2]. Let $L$ be a simple Lie algebra over an algebraically closed field $F$ of characteristic $p>7$. Let $T$ be an optimal torus in some p-envelope $\left(L_{p},[p], l\right)$ of $L$. Adopting the same notation as in Theorem 1.15 , we provide the following description of $\bar{T}$ for the various cases of that theorem.

(1) $\bar{T}=(0)$.

(2) $\bar{T}$ is conjugate under an automorphism of $\operatorname{Der} S$ to $\langle x d / d x\rangle$ if $S=$ $W(1: \underline{1})$ and to $\left\langle x^{\epsilon_{1}} D_{1}-x^{\epsilon_{2}} D_{2}\right\rangle$ if $S=H(2: \underline{\underline{1}})^{(2)}$.

(3) $\bar{T}=\left(\bar{T} \cap S_{1}\right) \oplus\left(\bar{T} \cap S_{2}\right)$ where $\bar{T} \cap S_{i}$ is as in (2). Moreover, there exist $\mathbb{Z}_{p}$-independent roots $\lambda, \mu \in \mathbb{Z}_{p} \alpha+\mathbb{Z}_{p} \beta$ such that $S_{1}=\pi\left(L^{(\lambda)}\right), S_{2}=\pi\left(L^{(\mu)}\right)$.

(4) $\bar{T}$ is conjugate under an automorphism of $\operatorname{Der} S$ to $\left\langle x^{\epsilon_{1}} D_{1}\right\rangle+\left\langle x^{\epsilon_{2}} D_{2}\right\rangle$.

(5) $\bar{T}$ leaves invariant $S \otimes\left(\sum_{i} x^{\epsilon_{i}} A(n: \underline{1})\right)$, and $\operatorname{dim} \bar{T} \cap(S \otimes A(n: \underline{1}))=1$.

(6) $\bar{T}=\left\langle t_{1}, t_{2}\right\rangle$ where $t_{1} \in S \otimes A(1: \underline{1}), t_{2} \in \mathrm{id}_{S} \otimes D_{1}+\operatorname{Nor}_{\bar{T}}(\operatorname{rad} K)$; and $\mu\left(C_{L}(T)\right)=0$ for some $\mu \in \mathbb{Z}_{p} \alpha+\mathbb{Z}_{p} \beta$.

(7) $\bar{T} \subset S_{p}$; and $\operatorname{dim} \bar{T} \cap S=q$ where $q=0$ if $S=H(2: \underline{\underline{1}}: \phi(\tau))^{(1)}$, and $q=1$ otherwise.

(8) $\bar{T} \subset S$.

Remark. In (7), $S_{p}$ denotes the $p$-subalgebra $\left\langle(\operatorname{ad} x)^{p^{r}} \mid x \in S, r=0,1, \ldots\right\rangle$ of Der $S$ generated by ad $S$. The fact that $q=1$ when $S=H(2: \underline{\underline{1}}: \Delta)$ will be established in Lemma 2.4 .

\section{1-SECTIONS IN 2-SECTIONS}

Our assumptions in this section are the following: $L$ is a finite-dimensional simple Lie algebra over an algebraically closed field $F$ of characteristic $p>7$; $T$ is an optimal torus in some $p$-envelope, $L_{p}$ of $L ; L^{(\alpha, \beta)}$ is a 2-section relative to $T ; I^{(\alpha, \beta)}$ is the maximal solvable ideal of $L^{(\alpha, \beta)}+T ; \pi: L^{(\alpha, \beta)}+$ $T \rightarrow\left(L^{(\alpha, \beta)}+T\right) / I^{(\alpha, \beta)}$ is the canonical projection; $K=\pi\left(L^{(\alpha, \beta)}\right) ; H=$ $C_{L}(T) ; \bar{H}=\pi(H) ;$ and $\bar{T}=\pi(T)$.

In this section we derive information about the 1-sections of $K$ relative to $\bar{T}$. Further information concerning the toral structure of $K$ can be found in [S3]. The analysis involves considering the various cases arising in Theorem 1.15. Since the 1 -sections of $K$ are quite easy to compute in the first three cases, we focus on (4)-(8). In examining the 1-sections, we need to perform calculations inside certain of the Cartan type Lie algebras. We let $S_{(0)}$ denote the standard maximal subalgebra of a Cartan type Lie algebra $S$, and $S=$ $S_{(-k)} \supset \cdots \supset S_{(-1)} \supset S_{(0)} \supset S_{(1)} \supset \cdots \supset S_{(l)}=(0)$, the standard filtration determined from $S_{(0)}$. The explicit basis used for $S$ will be that described in 
[SF, Chapter 4], where $\left\{x^{\underline{a}} \mid 0 \leq \underline{a} \leq\left(p^{n_{1}}-1, \ldots, p^{n_{m}}-1\right)=\tau(\underline{n})\right\}$ denotes the canonical basis for the divided power algebra $A(m: \underline{\underline{n}})$. We let $S_{p}$ denote the $p$-subalgebra $\left\langle(a d x)^{p^{r}} \mid x \in S, r=0,1, \ldots\right\rangle$ of Der $S$ generated by ad $S$. It will often be the case that $K \subseteq \operatorname{Der} S \subseteq \operatorname{ad} W(m: \underline{n}) \cong W(m: \underline{n})$ for some $m$ and $\underline{\underline{n}}$, and when that happens we will define $K_{(i)}=K \cap \operatorname{ad} \overline{\bar{W}}(m: \underline{\underline{n}})_{(i)}$ and $(\operatorname{Der} \overline{\bar{S}})_{(i)}=(\operatorname{Der} S) \cap \operatorname{ad} W(m: \underline{\underline{n}})_{(i)}$.

Before tackling the various cases we begin with a general result.

Lemma 2.1. Let $L^{(\zeta)}$ be a 1-section of $L^{(\alpha, \beta)}$ and $U^{(\zeta)}$ be its maximum compositionally classical subalgebra. Then $\operatorname{dim} \pi\left(L^{(\zeta)}\right) / \pi\left(U^{(\zeta)}\right)=\operatorname{dim} L^{(\zeta)} / U^{(\zeta)}$, and $\pi\left(U^{(\zeta)}\right)$ is the maximum compositionally classical subalgebra of $\pi\left(L^{(\zeta)}\right)$.

Proof. Observe first that $I^{(\alpha, \beta)} \cap U^{(\zeta)} \subseteq I^{(\alpha, \beta)} \cap L^{(\zeta)} \subseteq \operatorname{rad} L^{(\zeta)} \subseteq U^{(\zeta)}$. As a consequence, $I^{(\alpha, \beta)} \cap L^{(\zeta)}=I^{(\alpha, \beta)} \cap U^{(\bar{\zeta})}$. Then

$$
\pi\left(L^{(\zeta)}\right)=L^{(\zeta)} /\left(I^{(\alpha, \beta)} \cap U^{(\zeta)}\right)
$$

and

$$
\pi\left(U^{(\zeta)}\right)=U^{(\zeta)} /\left(I^{(\alpha, \beta)} \cap U^{(\zeta)}\right)
$$

It follows that $\pi\left(L^{(\zeta)}\right) / \pi\left(U^{(\zeta)}\right) \cong L^{(\zeta)} / U^{(\zeta)}$ (vector space isomorphism), to give the first assertion. Clearly any homomorphic image under $\pi$ of a compositionally classical subalgebra is a compositionally classical subalgebra. The second assertion is then clear from the first assertion and the definition of the maximum compositionally classical subalgebra.

Lemma 2.2 (Type 4). Assume that (4) of Theorem 1.15 holds so that $S:=$ $H\left(2: \frac{1}{\underline{2}}\right)^{(2)} \subseteq K+\bar{T} \subseteq \operatorname{Der} S$, and $\bar{T}$ is conjugate to $\left\langle x^{\epsilon_{1}} D_{1}\right\rangle+\left\langle x^{\epsilon_{2}} D_{2}\right\rangle$. Then there are two 1-sections which are Witt, one which is classical, and the remainder are solvable. The maximum compositionally classical subalgebra of $\pi\left(L^{(\zeta)}\right)$ is given by $\pi\left(U^{(\zeta)}\right)=K^{(\zeta)} \cap K_{(0)}$ for all roots $\zeta \in \mathbb{Z}_{p} \alpha+\mathbb{Z}_{p} \beta$.

Proof. By Proposition 2.1.8 of [BW4],

$$
\begin{aligned}
\operatorname{Der} S= & \left.\left\langle\operatorname{ad} \mathscr{D}\left(x^{\underline{a}}\right)\right| \underline{a}=\left(a_{1}, a_{2}\right) \neq(0,0) \text { and } 0 \leq \underline{a} \leq \tau(\underline{\underline{1}})\right\rangle \\
& \oplus\left\langle\operatorname{ad} \mathscr{D}\left(x^{p \epsilon_{i}}\right) \mid i=1,2\right\rangle \oplus\langle\operatorname{ad} \partial\rangle,
\end{aligned}
$$

where $\mathscr{D}(f)=\left(D_{1} f\right) D_{2}-\left(D_{2} f\right) D_{1}, \partial=x^{\epsilon_{1}} D_{1}+x^{\epsilon_{2}} D_{2}$, and

$$
\left[\mathscr{D}\left(x^{\underline{a}}\right), \mathscr{D}\left(x^{\underline{b}}\right)\right]=\left\{\left(\begin{array}{c}
\underline{a}+\underline{b}-\epsilon_{1}-\epsilon_{2} \\
\underline{a}-\epsilon_{1}
\end{array}\right)-\left(\begin{array}{c}
\underline{a}+\underline{b}-\epsilon_{1}-\epsilon_{2} \\
\underline{a}-\epsilon_{2}
\end{array}\right)\right\} \mathscr{D}\left(x^{\underline{a}+\underline{b}-\epsilon_{1}-\epsilon_{2}}\right) .
$$

Then

$$
\left[\partial, \mathscr{D}\left(x^{\underline{a}}\right)\right]=(|\underline{a}|-2) \mathscr{D}\left(x^{\underline{a}}\right),
$$

and for $\partial^{\prime}:=\mathscr{D}\left(x^{\epsilon_{1}+\epsilon_{2}}\right)=x^{\epsilon_{1}} D_{1}-x^{\epsilon_{2}} D_{2}$ we have

$$
\left[\partial^{\prime}, \mathscr{D}\left(x^{\underline{a}}\right)\right]=\left(a_{2}-a_{1}\right) \mathscr{D}\left(x^{\underline{a}}\right) .
$$

From these calculations it is clear that $\left\langle\mathscr{D}\left(x^{(j+1) \epsilon_{1}+\epsilon_{2}}\right) \mid j=-1, \ldots, p-2\right\rangle$ and $\left\langle\mathscr{D}\left(x^{\epsilon_{1}+(j+1) \epsilon_{2}}\right) \mid j=-1, \ldots, p-2\right\rangle$ determine 1-sections which are Witt and which correspond to multiples of the roots $(-1,1),(-1,-1)$, respectively, (where the pair gives the eigenvalues relative to ad $\partial$, ad $\partial^{\prime}$ ). The 1section containing $\left\langle\mathscr{D}\left(x^{2 \epsilon_{1}}\right), \mathscr{D}\left(x^{2 \epsilon_{2}}\right)\right\rangle$ corresponds to the roots which are multiples of $(0,2)$. The associated 1-section is classical. All other 1-sections lie in 
$S_{(1)}+\bar{T}+\left\langle\mathscr{D}\left(x^{(p-1) \epsilon_{1}+(p-1) \epsilon_{2}}\right)\right\rangle+\left\langle\mathscr{D}\left(x^{p \epsilon_{i}}\right) \mid i=1,2\right\rangle$, which is contained in $W(2: \underline{\underline{1}})_{(1)}+\bar{T}$, and so are solvable. The last assertion follows from the form of $\operatorname{Der} S$ and the calculations.

Lemma 2.3 (Type 5). Assume that case (5) of Theorem 1.15 holds so that $G:=$ $S \otimes A(n: \underline{\underline{1}}) \subseteq K+\bar{T} \subseteq \operatorname{Der} G$ where $S \in\left\{\operatorname{sl}(2), W(1: \underline{\underline{1}}), H(2: \underline{\underline{1}})^{(2)}\right\}$, $n=1$ or 2 , and the torus $\bar{T}$ leaves invariant $J:=S \otimes\left(\sum_{i} x^{\epsilon_{i}} A(n: \underline{\underline{1}})\right)$. Then there exist a root $\theta \neq 0$ and a nonzero toral element $t_{\theta} \in G \cap \operatorname{ker} \theta$ such that $K_{\theta} \not \subset(\operatorname{Der} S) \otimes A(n: \underline{\underline{1}})$, but for each root $\mu \notin \mathbb{Z}_{p} \theta, K_{\mu} \subset G$. Moreover, $\left[\pi\left(U^{(\theta)}\right), J\right] \subset J$. For some root $\eta \notin \mathbb{Z}_{p} \theta$ one of the following two cases occurs:

(1) $K^{(\eta)} / \operatorname{rad} K^{(\eta)} \supseteq S ; K^{(\eta+i \theta)}$ is solvable for all $i \neq 0$; and $K^{(\theta)}$ is Witt or Hamiltonian; or

(2) $S \cong H(2: \underline{1})^{(2)}$, and the induced action of $\bar{T}$ on $G / J \cong S$ is conjugate to the action of the torus $F \partial \oplus F \partial^{\prime}$ where $\partial=x^{\epsilon_{1}} D_{1}+x^{\epsilon_{2}} D_{2}$ and $\partial^{\prime}=x^{\epsilon_{1}} D_{1}-$ $x^{\epsilon_{2}} D_{2}$. The roots $\theta, \eta$ correspond to $(-1,0),(0,1)$, where the pair gives the eigenvalues relative to $\partial$ and $\partial^{\prime}$ respectively. The 1-sections $K^{(\eta-\theta)}$ and $K^{(\eta+\theta)}$ are Witt; $K^{(\eta)}$ is classical; $K^{(\theta)}$ is Witt or Hamiltonian; and all other 1-sections different from those are solvable.

Remark. A proof of this lemma could be assembled using [BW4, Lemmas 10.2.1, 11.2.5, and 5.8.5]. However, for the sake of completeness we have elected to include in this work another proof which is different from that given in [BW4], but uses many of the same ideas. Since our argument requires results on the other cases, we postpone the proof until after Lemma 2.6.

Lemma 2.4. (Type 6). Assume that case (6) of Theorem 1.15 holds so that $S \otimes A(1: \underline{\underline{1}}) \subseteq K+\bar{T} \subseteq \operatorname{Der}(S \otimes A(1: \underline{\underline{1}}))$ where $S \in\{$ sl(2), $W(1: \underline{\underline{1}})$, $\left.H(2: \underline{\underline{1}})^{(2)}\right\} ;$ and $(S \otimes A(1: \underline{\underline{1}})) \cap \operatorname{rad} K=S \otimes x A(1: \underline{\underline{1}})$. Then there exists $\mathbb{Z}_{p}$-independent roots $\eta, \theta \in \mathbb{Z}_{p} \alpha+\mathbb{Z}_{p} \beta$ such that $K^{(\eta+i \theta)} / \operatorname{rad} K^{(\eta+i \theta)} \supseteq S$ for $i=0, \ldots, p-1$ and $K^{(\theta)}$ is solvable. For each $j \neq 0$ there is an $x \in L_{j \theta}$ with $\eta\left(x^{[p]}\right) \neq 0$. Moreover, $K \subset(\operatorname{Der} S)^{(1)} \otimes A(1: \underline{\underline{1}})$ and for each root $\zeta$,

$$
\left.\pi\left(U^{(\zeta)}\right)=K^{(\zeta)} \cap\left\{(\operatorname{Der} S)_{(0)} \otimes F+(\operatorname{Der} S)^{(1)} \otimes x A(1: \underline{\underline{1}})\right)\right\} .
$$

Remark. The subalgebra $S_{(0)}$ is the maximum compositionally classical subalgebra of $S$. Thus,

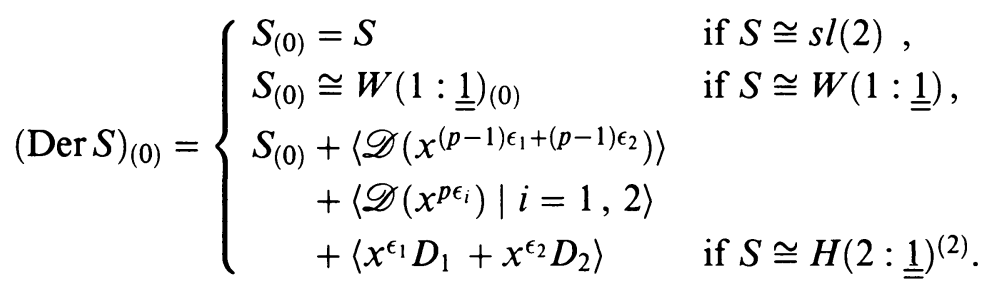

Proof. Theoremi IV.4 of [S3] shows that there are nonzero roots $\eta, \theta$ with $\eta(\bar{H}) \neq 0, \theta(\bar{H})=0$. Then $(\eta+i \theta)(\bar{H}) \neq 0$ for all $i \in \mathbb{Z}_{p}$, and $K^{(\theta)}$ is nilpotent by Theorem 1.5. We refer to [S3, Theorems IV.4, IV.5] to obtain the following:

(i) $K^{(\eta+i \theta)} \cap S \otimes A(1: \underline{\underline{1}}) / \operatorname{rad}\left(K^{(\eta+i \theta)} \cap S \otimes A(1: \underline{\underline{1}})\right) \cong S$ for every $i$;

(ii) for every $j \neq 0, K_{j \theta}$ contains an element $x$ with $\eta\left(x^{[p]}\right) \neq 0$;

(iii) $K \subseteq(\operatorname{Der} S)^{(1)} \otimes A(1: \underline{\underline{1}})$. 
Only the assertion on the compositionally classical subalgebra remains to be proved. If $S \cong s l(2)$, the assertion is clear. In the other cases consider the homomorphism

$$
\begin{aligned}
\phi:(\operatorname{Der} S)^{(1)} \otimes A(1: \underline{\underline{1}}) \longrightarrow(\operatorname{Der} S)^{(1)} \otimes A(1: \underline{\underline{1}}) /(\operatorname{Der} S)^{(1)} \otimes x A(1: \underline{\underline{1}}) \\
\\
\cong(\operatorname{Der} S)^{(1)} \otimes F \cong(\operatorname{Der} S)^{(1)} .
\end{aligned}
$$

In the argument to follow we identify $S, S \otimes F$, and $\operatorname{ad}_{S} S$. Take any root $\zeta \notin \mathbb{Z}_{p} \theta$, and observe that $S$ is a homomorphic image of $\phi\left(K^{(\zeta)} \cap S \otimes A(1: \underline{\underline{1}})\right)$ because of (i) above. The image is by the definition of $\phi$ contained in $S$, and thus $\phi\left(K^{(\zeta)} \cap S \otimes A(1: \underline{\underline{1}})\right)=S$ must hold. As $\zeta(\bar{H} \cap S \otimes A(1: \underline{\underline{1}})) \neq 0$, $\widehat{H}:=\phi(\bar{H} \cap S \otimes A(1: \underline{\underline{1}}))$ is a Cartan subalgebra of $S$ whose root spaces are given by

$$
S_{i \zeta}(\widehat{H})=\phi\left(\left(K^{(\zeta)} \cap S \otimes A(1: \underline{\underline{1}})\right)_{i \zeta}\right) .
$$

Thus, all roots with respect to $\widehat{H}$ are proper, and $\widehat{H}$ contains an element $\widehat{h}$ which is conjugate to $x D$ if $S \cong W(1: \underline{\underline{1}})$ or to $x^{\epsilon_{1}} D_{1}-x^{\epsilon_{2}} D_{2}$ if $S \cong$ $H\left(2: \underline{1}^{(2)}\right.$. Since $\theta(\bar{H})=0$, this implies that $\phi\left(K^{(\theta)}\right) \subset C_{(\operatorname{Der} S)^{(1)}}(\widehat{h}) \subset$ $(\operatorname{Der} S)_{(0)}$. Thus,

$$
K^{(\theta)} \subset(\operatorname{Der} S)_{(0)} \otimes F+(\operatorname{Der} S)^{(1)} \otimes x A(1: \underline{\underline{1}}),
$$

and since $\pi\left(U^{(\theta)}\right)=K^{(\theta)}$, the result is clear for the root $\theta$. When $\zeta \notin \mathbb{Z}_{p} \theta$, then $\phi\left(K^{(\zeta)}\right)=S+\phi(\bar{H}) \subset S+(\operatorname{Der} S)_{(0)}$. Thus, $S_{(0)}+\phi(\bar{H})$ is a maximal compositionally classical subalgebra of $\phi\left(K^{(\zeta)}\right)$, and so $\phi\left(\pi\left(U^{(\zeta)}\right)\right)=S_{(0)}+$ $\phi(\bar{H}) \subset(\operatorname{Der} S)_{(0)}$. Consequently,

$$
\pi\left(U^{(\zeta)}\right)=K^{(\zeta)} \cap\left\{(\operatorname{Der} S)_{(0)} \otimes F+(\operatorname{Der} S)^{(1)} \otimes x A(1: \underline{\underline{1}})\right\}
$$

as desired.

Lemma 2.5. (Type 7). Assume that case (7) of Theorem 1.15 holds, so that $\operatorname{Der} S \supseteq K+\bar{T} \supseteq S$ where

$$
S \in\left\{W(1: \underline{\underline{2}}), H(2:(2,1))^{(2)}, H(2: \underline{\underline{1}}: \phi(\tau))^{(1)}, H(2: \underline{\underline{1}}: \Delta)\right\} .
$$

Then the 1-sections of $K$ relative to $\bar{T}$ can be described as follows:

(i) $W(1: \underline{\underline{2}})\left(H(2:(2,1))^{(2)}\right)$ : There exists $\mathbb{Z}_{p}$-independent roots $\eta, \theta \in$ $\mathbb{Z}_{p} \alpha+\mathbb{Z}_{p} \beta$ such that $K^{(\eta+i \theta)}$ is Witt (resp. Hamiltonian) for $i=0, \ldots, p-1$ and $K^{(\theta)}$ is solvable.

(ii) $H(2: \underline{1}: \phi(\tau))^{(1)}:$ all 1-sections solvable.

(iii) $H(2: \underline{\underline{1}}: \Delta):$ two Witt, one classical, all others solvable.

The maximum compositionally classical subalgebra of $K^{(\zeta)}$ is given by $\pi\left(U^{(\zeta)}\right)=$ $K^{(\zeta)} \cap S_{(0)}$ for all roots $\zeta \in \mathbb{Z}_{p} \alpha+\mathbb{Z}_{p} \beta$ in (i). In case (ii), $\pi\left(U^{(\zeta)}\right)=K^{(\zeta)}$ for all roots $\zeta$. And in (iii) $\pi\left(U^{(\zeta)}\right)=K^{(\zeta)} \cap K_{(0)}$ for all roots $\zeta$.

Proof. By Theorem 1.16, $\bar{T} \subset S_{p}$. As a consequence, all root vectors relative to $\bar{T}$ for nonzero roots belong to $S$, hence to $K$. We make use of these results in our deliberations.

(i) $W(1: \underline{\underline{2}}), H(2:(2,1))^{(2)}:$ First let $S=W(1: \underline{\underline{2}})$. Then $S \subseteq K+\bar{T} \subseteq$ $\operatorname{Der} S=S_{p}=S+\left\langle D^{p}\right\rangle$, and $\operatorname{dim} \bar{T} \cap S=1$ by Theorem 1.16. Since $\operatorname{dim} \bar{T}=2$, 
it must be that $S+\bar{T}=K+\bar{T}=S_{p}$. Lemma 11.1.1 of [BW4] applies. In particular, it follows from that lemma that if $t_{\zeta} \in \bar{T}$ is a toral element in the kernel of the root $\zeta$, then $t_{\zeta} \notin S$ implies that the 1-section $S_{p}^{(\zeta)}(\bar{T})$ is Witt. While if $t_{\zeta} \in S$, then $S_{p}^{(\zeta)}(\bar{T})$ is solvable. Moreover, part (d) of that lemma shows that $t_{\theta} \in S$ for some root $\theta$, whenever all roots relative to $\bar{T}$ are proper. Since $\operatorname{dim} T \cap S=1, t_{\zeta} \notin S$ for any root $\zeta$ which is $\mathbb{Z}_{p}$-independent of $\theta$. In particular then, such a root $\zeta$ is Witt.

Suppose now that $t_{\theta} \in S$, say $t_{\theta} \equiv a D_{1}$ modulo $S_{(0)}$. Then $t_{\theta}=t_{\theta}^{p} \equiv a^{p} D_{1}^{p}$ modulo $S$, showing that $a=0$. Thus, it must be that $t_{\theta} \in S_{(0)}$, and because $t_{\theta}$ is toral, $t_{\theta} \notin S_{(1)}$. Since $t_{\theta} \equiv c x^{\epsilon_{1}} D_{1}$ modulo $S_{(1)}$ for some $c \neq 0$, we see that $C:=C_{S}\left(t_{\theta}\right) \subset C_{\text {Der } S}\left(t_{\theta}\right) \subset(\operatorname{Der} S)_{(0)}$. Let $T^{\prime}=\left\langle t_{\theta}\right\rangle$. There is a root $\eta \notin \mathbb{Z}_{p} \theta$ of $\bar{T}$ such that if $\tilde{\eta}$ is defined on $T^{\prime}$ by $\tilde{\eta}\left(t_{\theta}\right)=\eta\left(t_{\theta}\right)$, then roots relative to $T^{\prime}$ are multiples of $\tilde{\eta}$. Moreover we may assume that $\eta$ is chosen so that on $S_{(-1)} / S_{(0)}, T^{\prime}$ has root $-\tilde{\eta}$. Then $S_{j \tilde{\eta}}\left(T^{\prime}\right)=\sum_{r \in \mathbb{Z}_{p}} S_{j \eta+r \theta}(\bar{T})$ is contained in $S_{(0)}$ whenever $j \neq-1$. When $j=-1$, its intersection with $S_{(0)}$ has codimension one in it. Part (d) of [BW, Lemma 11.1.1] proves the existence of an element $x \in L_{\theta}$ such that $x^{[p]}$ is not nil. Then $L_{\eta+i \theta}=(\operatorname{ad} x)^{i}\left(L_{\eta}\right) \neq(0)$, and all $\eta+i \theta$ are roots, necessarily of Witt type. If $\theta(H) \neq 0$, then there would be a root $\gamma$ such that $\theta\left(\left[L_{\gamma}, L_{-\gamma}\right]\right) \neq 0$ and $\gamma\left(x^{[p]}\right) \neq 0$. Let $M$ denote the $p$-envelope of $L^{(\gamma, \theta)}$. Then Lemma 11.2.1 of [BW4] applies to $M$ to give a contradiction. Thus, $\theta(H)=0$, and in particular $T \not \subset L$, proving that $K \neq S_{p}$. Hence, $K=S$, so $C=C_{K}\left(t_{\theta}\right)=C_{S}\left(t_{\theta}\right) \subset S_{(0)}$.

The situation for $S=H(2:(2,1))^{(2)}$ is similar. By Proposition 2.1.8 of [BW4], $S_{p}=S+\left\langle\left(\operatorname{ad} D_{1}\right)^{p}\right\rangle$, and by Theorem $1.16, \operatorname{dim} \bar{T} \cap S=1$. Since $S_{p}$ contains no torus of dimension $>2$, we may apply Lemma 10.1.1 of [BW4] to the algebra $S_{p}$. In particular, if $t_{\zeta}$ denotes a toral element in the kernel of the root $\zeta$, then $t_{\zeta} \notin S$ implies that $S_{p}^{(\zeta)}(\bar{T})$ is Hamiltonian, while $t_{\zeta} \in S$ implies that $S_{p}^{(\zeta)}(\bar{T})$ is solvable. Whenever all roots are proper, there must exist some root $\theta$ with $S_{p}^{(\theta)}(\bar{T})$ solvable and $T \cap S_{(0)} \neq(0)$ by parts (f) and (d) of that lemma. Since $\operatorname{dim} \bar{T} \cap S=1, t_{\theta} \in S_{(0)}$, as in the case just done. Moreover, $t_{\zeta} \notin S$ for any root $\zeta \notin \mathbb{Z}_{p} \theta$, and such a root $\zeta$ is Hamiltonian. Then $T^{\prime}=\left\langle t_{\theta}\right\rangle$ is a torus of $S_{(0)}$, and $T^{\prime}+S_{(1)} / S_{(1)}$ is a Cartan subalgebra of $S_{(0)} / S_{(1)} \cong s l(2)$. Its roots on the 2-dimensional module $S_{(-1)} / S_{(0)}$ are opposites. Thus, there exists a root $\eta \notin \mathbb{Z}_{p} \theta$ such that if $\tilde{\eta}\left(t_{\theta}\right):=\eta\left(t_{\theta}\right)$, then the roots of $T^{\prime}$ on $S_{(-1)} / S_{(0)}$ are $\pm \tilde{\eta}$. Roots of $S$ relative to $T^{\prime}$ are multiples of $\tilde{\eta}$. Now $\sum_{r \in \mathbb{Z}_{p}} S^{j \eta+r \theta}(\bar{T})=S^{j \tilde{\eta}}\left(T^{\prime}\right)$, and whenever $j \neq 1,-1$, this space is contained in $S_{(0)}$. When $j=1$ or -1 its intersection with $S_{(0)}$ has codimension one in it, and similar to the previous case we have $K=S$.

When $S=W(1: \underline{\underline{2}})$ or $H(2:(2,1))^{(2)}$, then $K^{(\theta)} \subset C_{S}\left(t_{\theta}\right) \subset S_{(0)}$ to give the assertion about $\pi\left(U^{(\theta)}\right)=K^{(\theta)}$. Suppose now that $\zeta=\eta+i \theta$, so that $K^{(\zeta)}=$ $K^{(\zeta)} \cap S+\bar{H}$. By our previous calculations, $K^{(\zeta)} \cap S_{(0)}$ has codimension one in $S$ if $\zeta$ is Witt and codimension two if $\zeta$ is Hamiltonian. Then $K^{(\zeta)} \cap(\operatorname{Der} S)_{(0)}$ has the same codimension in $K^{(\zeta)}$. As $S_{(0)}$ is compositionally classical, $K^{(\zeta)} \cap$ $S_{(0)}$ is the maximum compositionally classical subalgebra of $K^{(\zeta)}$.

(ii) $H(2: \underline{\underline{1}}: \phi(\tau))^{(1)}$ : We quote Lemma 11.1.2 of [BW4] to obtain: $S_{p}^{(\zeta)}(\bar{T})$ is solvable for all roots $\zeta ; C_{S_{p}}(\bar{T})=\bar{T}$; and $\bar{T} \cap S=(0)$. Since $K^{(\zeta)}(\bar{T})=$ 
$S^{(\zeta)}(\bar{T})+C_{K}(\bar{T})$, where $S^{(\zeta)}(\bar{T})$ is solvable, and $C_{K}(\bar{T})$ is nilpotent, it musi be that all 1 -sections of $K$ relative to $\bar{T}$ are solvable.

(iii) $H(2: \underline{\underline{1}}: \Delta)$ : By Proposition 2.1 .8 of [BW4], Der $S=S_{p}=S+$ $\left\langle\partial:=x^{\epsilon_{1}} D_{1}+\bar{x}^{\epsilon_{2}} D_{2}\right\rangle$. Hence, $S \subseteq K+\bar{T} \subseteq S+\langle\partial\rangle$. We determine from Lemma 11.1 .3 of [BW4] that $\bar{T} \subset\left(S_{p}\right)_{(0)}=S_{(0)}+\langle\partial\rangle$, and $S_{p}^{(\zeta)}(\bar{T})$ is Witt whenever $S_{p}^{(\zeta)}(\bar{T}) \nsubseteq\left(S_{p}\right)_{(0)}$. Then $T_{0}:=\bar{T} \cap S_{(0)}$ is a torus of $S_{(0)}$. Therefore, $T_{0}+S_{(1)} / S_{(1)}$ is a Cartan subalgebra of $S_{(0)} / S_{(1)} \cong \operatorname{sl}(2), \operatorname{dim} T_{0}=1, \bar{T}=$ $T_{0}+\langle\partial\rangle$, and $S_{(-1)} / S_{(0)}$ is the natural 2-dimensional representation of $S_{(0)} / S_{(1)}$ on $S_{(-1)} / S_{(0)}$. Thus, there are two different roots relative to $T_{0}$ on $S_{(-1)} / S_{(0)}$. Since ad $\partial$ leaves $S_{(0)}$ invariant, there are exactly two root spaces of $S_{p}$ relative to $\bar{T}$ which do not lie in $S_{(0)}$. They determine different 1 -sections, because the value of $\partial$ on them is -1 , while the values of a toral element $t_{0} \in T_{0}$ may be taken to be -1 and 1 . Thus, there are precisely two 1 -sections which are Witt. On $S_{(0)} / S_{(1)}, t_{0}$ assumes values $2,0,-2$, and $\partial$ value 0 . Thus, the 1 -section determined by multiples of the root $(2,0)$ is classical. The remaining 1 -sections belong to $S_{(1)}+\bar{T}$ and so are solvable. If $\zeta$ is a Witt root, then $K^{(\zeta)} \cap K_{(0)}$ is compositionally classical and has codimension one in $K^{(\zeta)}$. Hence, it must be $\pi\left(U^{(\zeta)}\right)$. For the other roots $\pi\left(U^{(\zeta)}\right)=K^{(\zeta)} \cap K_{(0)}$ clearly holds.

Lemma 2.6 (Type 8). Assume that case (8) of Theorem 1.15 holds, so that $\operatorname{Der} S \supset K=S+C_{K}(\bar{T})$ where $S \in\left\{A_{2}, C_{2}, G_{2}, W(2: \underline{\underline{1}}), S(3: \underline{\underline{1}})^{(1)}\right.$, $\left.H(4: \underline{1})^{(2)}, K(3: \underline{1})\right\}$, and $\bar{T} \subset S$. Then the 1 -sections of $K$ relative to $\overline{\bar{T}}$ are the following.

(i) $A_{2}, C_{2}, G_{2}$ : all classical, and the number of them is 3, 4, or 6, respectively.

(ii) $W(2: \underline{1})$ : two Witt, one classical, all others solvable.

(iii) $S(3: \underline{\underline{1}})^{(1)}$ : three Witt, three classical, all others solvable.

(iv) $H(4: \underline{1})^{(2)}$ : two Hamiltonian, two classical, all others solvable.

(v) $K(3: \underline{1})$ : three Witt, one classical, all others solvable.

Moreover, the maximum compositionally classical subalgebra of $K^{(\zeta)}=\pi\left(L^{(\zeta)}\right)$ is given by $\pi\left(U^{(\zeta)}\right)=K^{(\zeta)} \cap K_{(0)}$ for all roots $\zeta \in \mathbb{Z}_{p} \alpha+\mathbb{Z}_{p} \beta$ whenever $S$ is nonclassical.

Proof. The assertions in (i) are clear from the classical theory. In the remaining cases, since $\bar{T} \subset S$ and $K=S+C_{K}(\bar{T})$, all root vectors for nonzero roots relative to $\bar{T}$ belong to $S$. Because roots relative to $\bar{T}$ are proper, it follows from [BW4, Lemma 5.8.2(h)], that $\bar{T}$ is conjugate under the automorphism group of $S$ to the standard maximal torus $T_{0}$ inside the maximal subalgebra $S_{(0)}$. Hence, it suffices to examine the 1-sections of $S$ relative to the standard maximal torus $T_{0}$. Since $S_{(1)}+\bar{H}$ is solvable, every 1 -section having all its root vectors in $S_{(1)}+\bar{H}$ is solvable.

(ii) $S=W(2: \underline{\underline{1}})=\left\langle x \underline{\underline{r}} D_{j} \mid \underline{r}=\left(r_{1}, r_{2}\right), 0 \leq r_{i} \leq p-1, j=1,2\right\rangle$, and $T_{0}=\left\langle x^{\epsilon_{1}} D_{1}, x^{\epsilon_{2}} D_{2}\right\rangle$. The elements $x^{r} D_{j}$ are eigenvectors relative to ad $T_{0}$. It is easy to check that $\left\langle D_{j}\right\rangle \subset\left\langle x^{s \epsilon_{j}} D_{j} \mid s=0, \ldots, p-1\right\rangle \cong W(1: \underline{\underline{1}})$ for $j=1,2$. The elements $x^{\epsilon_{1}} D_{2}, x^{\epsilon_{2}} D_{1}$ belong to a 1-section which is classical. Since the root vectors of all other 1-sections relative to $T_{0}$ lie in $S_{(1)}$, the other 1-sections are solvable. Thus, the 1 -sections of $K$ are as asserted. As $\operatorname{Der} S=\operatorname{ad} S \cong S$, it is clear from the calculations that $K^{(\zeta)} \cap S_{(0)}$ is compositionally classical and 
has codimension 1 whenever $\zeta$ is Witt. By Proposition 1.11 and Lemma 2.1, it must be $\pi\left(U^{(\zeta)}\right)$.

(iii) $S=S(3: \underline{\underline{1}})^{(1)}$ is spanned by $\left\{D_{i, j}\left(x^{\underline{r}}\right) \mid x^{\underline{r}} \in A(3: \underline{\underline{1}}), 1 \leq i<j \leq 3\right\}$ where $D_{i, j}\left(x^{\underline{r}}\right)=D_{j}\left(x^{\underline{r}}\right) D_{i}-D_{i}\left(x^{\underline{r}}\right) D_{j}$. Then

$$
T_{0}=\left\langle D_{k, l}\left(x^{\epsilon_{k}+\epsilon_{l}}\right)=x^{\epsilon_{k}} D_{k}-x^{\epsilon_{l}} D_{l} \mid 1 \leq k<l \leq 3\right\rangle .
$$

Since

$$
\left[D_{k, l}\left(x^{\epsilon_{k}+\epsilon_{l}}\right), D_{i, j}\left(x^{\underline{r}}\right)\right]=\left(r_{k}-\delta_{k, i}-\delta_{k, j}-r_{l}+\delta_{l, i}+\delta_{l, j}\right) D_{i, j}\left(x^{\underline{r}}\right),
$$

the elements $D_{i, j}\left(x^{\underline{r}}\right)$ are eigenvectors relative to ad $T_{0}$. The 1-section containing $\left\langle D_{i}\right\rangle \subset\left\langle D_{i, j}\left(x^{s \epsilon_{i}+\epsilon_{j}}\right) \mid 0 \leq s \leq p-1\right\rangle$ is Witt, and there are three such 1sections. For $i, j, k$ distinct, the elements $\left\{D_{i, j}\left(x^{\epsilon_{j}+\epsilon_{k}}\right)=x^{\epsilon_{k}} D_{i}, D_{k, j}\left(x^{\epsilon_{i}+\epsilon_{j}}\right)\right.$ $\left.=x^{\epsilon_{i}} D_{k}\right\}$ belong to a 1-section which is classical. As in (ii) above, the remaining 1-sections are solvable. Now in this case $K \subseteq \operatorname{Der} S=\operatorname{ad} S(3: \underline{\underline{1}})+\operatorname{ad} x^{\epsilon_{1}} D_{1}$ where $S(3: \underline{\underline{1}})=\left\{\sum_{i=1}^{3} f_{i} D_{i} \mid \sum_{i=1}^{3} D_{i}\left(f_{i}\right)=0\right\}$. Since $S(3: \underline{1})_{(-1)}$ is spanned modulo $S(3: \underline{\underline{1}})_{(0)}$ by $D_{1}, D_{2}, D_{3}$, (see [SF, Proposition 4.3.3]), the assertion that $K^{(\zeta)} \cap K_{(0)}=\pi\left(U^{(\zeta)}\right)$ is clear.

(iv) $S=H(4: \underline{\underline{1}})^{(2)}=\left\langle\mathscr{D}\left(x^{\underline{r}}\right) \mid x^{\underline{r}} \in A(4: \underline{\underline{1}}), 0<\underline{r}<\tau(\underline{\underline{1}})\right\rangle$, in the notation of [SF, §4.4]. Then $T_{0}=\left\langle\mathscr{D}\left(x^{\epsilon_{1}+\epsilon_{3}}\right), \mathscr{D}\left(x^{\epsilon_{2}+\epsilon_{4}}\right)\right\rangle$. The two 1-sections $\left\langle\mathscr{D}\left(x^{\left.r \epsilon_{1}+s \epsilon_{3}\right)}|(r, s) \neq(0,0)\rangle+C_{K}\left(T_{0}\right),\left\langle\mathscr{D}\left(x^{\left.r \epsilon_{2}+s \epsilon_{4}\right)}|(r, s) \neq(0,0)\rangle+\right.\right.\right.\right.$ $C_{K}\left(T_{0}\right)$ which contain $\left\langle D_{1}, D_{3}\right\rangle,\left\langle D_{1}, D_{4}\right\rangle$ respectively are Hamiltonian. The 1-sections which contain $\left\langle\mathscr{D}\left(x^{\epsilon_{1}+\epsilon_{2}}\right), \mathscr{D}\left(x^{\epsilon_{3}+\epsilon_{4}}\right)\right\rangle$ or $\left\langle\mathscr{D}\left(x^{\epsilon_{1}+\epsilon_{4}}\right), \mathscr{D}\left(x^{\epsilon_{2}+\epsilon_{3}}\right)\right\rangle$ are classical, and the remainder are solvable. Now

$$
\operatorname{Der} S=\left\langle\operatorname{ad} \mathscr{D}\left(x^{\underline{r}}\right) \mid \underline{r} \neq(0,0,0,0)\right\rangle \oplus\left\langle\operatorname{ad} \mathscr{D}\left(x^{p \epsilon_{i}}\right) \mid i=1, \ldots, 4\right\rangle \oplus\langle\operatorname{ad} \partial\rangle,
$$

where $\mathscr{D}\left(x^{\underline{r}}\right) \in W(4: \underline{\underline{1}})_{|\underline{r}|-2}$ and $\partial=\sum_{i=1}^{4} x^{\epsilon_{i}} D_{i}$. Thus, it is apparent that $K^{(\zeta)} \cap(\operatorname{Der} S)_{(0)}$ (which is $K^{(\zeta)} \cap K_{(0)}$ ) is compositionally classical and has codimension $\leq 2$. It must then be $\pi\left(U^{(\zeta)}\right)$ by Lemma 2.1 .

(v) $S=K(3: \underline{\underline{1}})$. Then $S=\left\langle x^{\underline{r}} \mid x^{\underline{r}} \in A(3: \underline{\underline{1}})\right\rangle$, as discussed in [SF, §4.5], and multiplication in $S$ is given by

$$
\prec x^{\underline{r}}, x^{\underline{s}} \succ=\left\{x^{\underline{r}}, x^{\underline{s}}\right\}+\left(\|\underline{s}\|\left(\begin{array}{c}
\underline{r}+\underline{s}-\epsilon_{3} \\
\underline{s}
\end{array}\right)-\|\underline{r}\|\left(\begin{array}{c}
\underline{r}+\underline{s}-\epsilon_{3} \\
\underline{r}
\end{array}\right)\right) x^{\underline{r}+\underline{s}-\epsilon_{3}}
$$

where $\{f, g\}=D_{1}(f) D_{2}(g)-D_{2}(f) D_{1}(g)$ and $\|\underline{r}\|=|\underline{r}|+r_{3}-2$. The torus $T_{0}$ is spanned by the two toral elements $x^{\epsilon_{1}+\epsilon_{2}}, x^{\epsilon_{3}}$, where $\prec x^{\epsilon_{1}+\epsilon_{2}}, x^{\underline{s}} \succ=$ $\left(s_{2}-s_{1}\right) x^{\underline{s}}$ and $\prec x^{\epsilon_{3}}, x^{\underline{s}} \succ=\|\underline{s}\| x \underline{s}$. We denote roots by their values on the two toral elements. The 1-section containing $\left\langle x^{\epsilon_{2}}\right\rangle \subset\left\langle x^{s \epsilon_{1}+\epsilon_{2}} \mid s=0, \ldots, p-1\right\rangle$ corresponds to roots which are $\mathbb{Z}_{p}$-multiples of $(1,-1)$. Similarly, the 1-section containing $\left\langle x^{\epsilon_{1}}\right\rangle \subset\left\langle x^{\epsilon_{1}+s \epsilon_{2}} \mid s=0, \ldots, p-1\right\rangle$ corresponds to roots which are multiples of $(-1,-1)$, and $\langle 1\rangle \subset\left\langle x^{s \epsilon_{3}} \mid s=0, \ldots, p-1\right\rangle$ to roots that are multiples $(0,1)$. These elements determine three different 1 -sections, each of which is Witt. The 1-section corresponding to the root $(1,0)$, to which $x^{2 \epsilon_{1}}$ and $x^{2 \epsilon_{2}}$ belong, is classical, and the others solvable. In this case, $\operatorname{Der} S=S$ as shown in [SF, Theorem 4.8.8], so the remaining assertion is clear. This completes this case and the lemma.

Proof of Lemma 2.3. Let $G=S \otimes A(n: \underline{\underline{1}})$ and $J=S \otimes\left(\sum_{i} x^{\epsilon_{i}} A(n: \underline{\underline{1}})\right)$ as in the statement of the theorem, and let $T^{\prime}=\bar{T} \cap G$. Then $\operatorname{dim} T^{\prime}=1$ 
by Theorem 1.16. If $t \in T^{\prime}$, then $t^{[p]} \in \bar{T} \cap G=T^{\prime}$ as $G$ is restricted. Thus, $T^{\prime}$ is a torus of $G$ and is spanned by some toral element. We can find $\theta \in \mathbb{Z}_{p} \alpha+\mathbb{Z}_{p} \beta$ with $\theta\left(T^{\prime}\right)=0$. Let $t_{\theta} \in T^{\prime}$ be a toral element and consider the induced action of $\bar{T}$ on $G / J \cong S$. Either (1) there is a root $\eta$ with $\eta\left(t_{\theta}\right) \neq 0$ such that the roots of $\bar{T}$ on $G / J$ are multiples of $\eta$ (this is necessarily the case if $S \cong s l(2)$ or $W(1: \underline{1}))$, or else (2) $S \cong H(2: \underline{1})^{(2)}$, and the induced action of $\bar{T}$ on $G / J \cong H(2: \underline{1})^{(2)}$ is that described in Lemma 2.2. Since $G$ is an ideal of $K+\bar{T}$ and $t_{\theta} \in G$, any root space of $K$ outside of $G$ must correspond to a multiple of $\theta$. From this discussion we conclude in case (1) that $K^{(\eta)} / \operatorname{rad} K^{(\eta)} \supseteq S$, and $K^{(\eta+i \theta)} \subseteq J+C_{K}(\bar{T})$, and so is solvable for all $i \neq 0$. Moreover the portion of $\sum_{j=1}^{p-1} K_{j \theta}$ lying in $G$ in fact lies in $J$. In case (2) all assertions except for the fact that $K^{(\theta)}$ is Witt or Hamiltonian can be verified precisely as in the proof of Lemma 2.2 .

What remains to be shown in both case (1) and case (2) is that $K^{(\theta)}$ is Witt or Hamiltonian and that $U^{(\theta)}$ normalizes $J$. Observe that $J$ is the unique maximal ideal of $G$, and it is nilpotent and $\bar{T}$-invariant. For any root vector $y \in L_{i \theta}$, let $\bar{y}:=\pi(y)$ and consider

$$
I_{\bar{y}}:=\sum_{k \geq 0}(\operatorname{ad} \bar{y})^{k}(J) .
$$

Since $[\bar{z}, \bar{y}] \in G$ for all $\bar{z} \in G$ and since $J$ is an ideal of $G, I_{\bar{y}}$ is an ideal of $G$ which contains $J$ and is $\bar{T}$-invariant. If $I_{\bar{y}} \subset J$ for all $\bar{y} \in \bigcup_{i \in \mathbb{Z}_{p}} K_{i \theta}$, then $\left[K^{(\theta)}, J\right] \subset J$. As $J$ is $\bar{T}$-invariant and an ideal of $G$, it would be a nilpotent ideal of $K+\bar{T}$, which is impossible. Thus, there is an $i \in \mathbb{Z}_{p}$ and $y \in L_{i \theta}$ such that $I:=I_{\bar{y}}$ is not contained in $J$. The maximality of $J$ shows that $I=G$. It follows that

$$
\bar{H} \cap G=\bar{H} \cap J+\sum_{k \geq 1}(\operatorname{ad} \bar{y})^{k} J_{-k i \theta} .
$$

If $i=0$, then $\bar{H} \cap G \subset \bar{H} \cap J+\bar{H}^{(1)}$ and $\bar{H} \cap G$ would act nilpotently on $G$. This contradiction shows that $i \neq 0$, and we may replace $\theta$ by an appropriate multiple if necessary to obtain $y \in L_{\theta}$. Observe that $\bar{y} \notin(\operatorname{Der} S) \otimes A(n: \underline{1})$ since $J$ is not invariant under ad $\bar{y}$.

Now $K_{\theta} \cap G$ is an ideal of $K^{(\theta)}$ (as $G$ is an ideal of $K$ ). Since no nonzero multiple of $\theta$ is a root of $\bar{T}$ on $G / J$, it must be that $K^{(\theta)} \cap G \subset J+\bar{H}$. Thus, $K^{(\theta)} \cap G$ is solvable and $K^{(\theta)} \cap G \subset \operatorname{rad} K^{(\theta)}$. Now as $J_{-\theta} \subset \operatorname{rad} K^{(\theta)}$, we have

$$
\Phi:=\left\{\lambda \mid \lambda\left(\left[y,\left(\operatorname{rad} L^{(\theta)}\right)_{-\theta}\right]\right) \neq 0\right\} \neq \varnothing .
$$

Schue's Lemma (see, for example, [BW4, Lemma 1.12.1] implies that

$$
H=\sum_{\lambda \in \Phi}\left[L_{\lambda}, L_{-\lambda}\right]
$$

Since we are in case $(5), \theta(H) \neq 0$. Thus there is a $\lambda$ with

$$
\lambda\left(\left[y,\left(\operatorname{rad} L^{(\theta)}\right)_{-\theta}\right]\right) \neq 0, \quad \text { and } \theta\left(\left[L_{\lambda}, L_{-\lambda}\right]\right) \neq 0 .
$$

Let $M$ be any $T$-invariant ideal of the 2 -section $L^{(\lambda, \theta)}$. Assume for some choice of $(r, s) \neq(0,0), r, s \in \mathbb{Z}_{p}$ that

$$
(r \theta+s \lambda)(H \cap M)=0 .
$$


If $\theta(H \cap M) \neq 0$, then $L_{\theta},\left(\operatorname{rad} L^{(\theta)}\right)_{-\theta} \subset\left[L^{(\theta)}, H \cap M\right] \subset M$. As

$$
\theta\left(\left[L_{\theta},\left(\operatorname{rad} L^{(\theta)}\right)_{-\theta}\right]\right)=0,
$$

we obtain from our assumption that

$$
0=(r \theta+s \lambda)\left(\left[L_{\theta},\left(\operatorname{rad} L^{(\theta)}\right)_{-\theta}\right]\right)=s \lambda\left(\left[L_{\theta},\left(\operatorname{rad} L^{(\theta)}\right)_{-\theta}\right]\right) .
$$

Then $(*)$ implies that $s=0$. Therefore, $r \theta(H \cap M)=0$, and as $\theta(H \cap M) \neq$ 0 we obtain the contradiction $(r, s)=(0,0)$. Thus, $\theta(H \cap M)=0$ must hold. Similarly, if $\lambda(H \cap M) \neq 0$, then $L_{\lambda}, L_{-\lambda} \subset\left[L^{(\lambda)}, H \cap M\right] \subset M$, and $\left[L_{\lambda}, L_{-\lambda}\right] \subset H \cap M$. Then $\theta\left(\left[L_{\lambda}, L_{-\lambda}\right]\right) \neq 0$ implies $\theta(H \cap M) \neq 0$, a contradiction. Thus, the above assumption implies that $\theta(H \cap M)=0$, $\lambda(H \cap M)=0$, and hence that $(i \theta+j \lambda)(H \cap M)=0$ for all $i, j$.

Now it follows from $(*)$ that

$$
L_{j \theta}=\left[L_{j \theta},\left[L_{\lambda}, L_{-\lambda}\right]\right] \text { and } L_{j \lambda}=\left[L_{j \lambda},\left[L_{\theta}, L_{-\theta}\right]\right]
$$

for all $j \neq 0$. Thus, $L^{(\theta, \lambda)}$ is nonsolvable, and hence not of type (1). If $L^{(\theta, \lambda)}$ is of type (2)-(7), there is an $T$-invariant ideal $M$ of $L^{(\theta, \lambda)}$ such that $H \cap M$ vanishes on one root $r \theta+s \lambda$, but not all linear combinations of $\theta$ and $\lambda$. We conclude from the argument of the previous paragraph that $L^{(\theta, \lambda)}$ must be of type (8).

Suppose first that $\left(L^{(\theta, \lambda)}+T\right) / I^{(\theta, \lambda)} \supset S^{\prime}$ where the unique minimal ideal $S^{\prime}$ is classical. Then $\left(\operatorname{rad} L^{(\theta)}\right)_{-\theta}$ maps to 0 in this quotient algebra, which contradicts $(*)$. We may assume then that $S^{\prime}$ is nonclassical. If $\theta$ is solvable or classical, then $\pi_{\theta, \lambda}\left(L^{(\theta)}\right) \subset\left(\operatorname{Der} S^{\prime}\right)_{(0)}$. As $\left(\operatorname{Der} S^{\prime}\right)_{(0)} /\left(\operatorname{Der} S^{\prime}\right)_{(1)}$ is classical reductive, we obtain that $\left(\operatorname{rad} L^{(\theta)}\right)_{-\theta}$ maps into $\left(\operatorname{Der} S^{\prime}\right)_{(1)}$, which implies that $\pi_{\theta, \lambda}\left(\left[L_{\theta},\left(\operatorname{rad} L^{(\theta)}\right)_{-\theta}\right]\right) \subset\left(\operatorname{Der} S^{\prime}\right)_{(1)}$. This contradicts $(*)$. Consequently, $\theta$ must be Witt or Hamiltonian, and $\pi_{\theta, \lambda}\left(\operatorname{rad} L^{(\theta)}\right) \subset \pi_{\theta, \lambda}(H)+\left(\operatorname{Der} S^{\prime}\right)_{(1)}$. Since according to $(*),\left[y,\left(\operatorname{rad} L^{(\theta)}\right)_{-\theta}\right]$ is not mapped into $\left(\operatorname{Der} S^{\prime}\right)_{(1)}, \pi_{\theta, \lambda}(y)$ cannot be contained in $\left(\operatorname{Der} S^{\prime}\right)_{(0)}$. Thus, $y \notin U^{(\theta)}$. This completes the proof of the lemma.

We conclude this section with a corollary to the lemma just proven giving more detailed information for 2-sections of type (5).

Corollary 2.7. Assume that the hypotheses of Lemma 2.3 hold and let $\theta, \eta, G$, and $J$ be as in that lemma. Let $M:=(\operatorname{Der} S) \otimes A(n: \underline{\underline{1}})$ so that the normalizer of $J$ in $\operatorname{Der} G$ is given by $N:=M \oplus \operatorname{id}_{S} \otimes W(n: \underline{\underline{1}})_{(0)}$. Then $K^{(\eta+i \theta)} \subset G+\bar{H}$ for all $i$

$$
\bar{H} \subset N_{0}:=(\operatorname{Der} S)_{(0)} \otimes F+(\operatorname{Der} S) \otimes\left(\sum_{i} x^{\epsilon_{i}} A(n: \underline{\underline{1}})\right)+\operatorname{id}_{S} \otimes W(n: \underline{\underline{1}})_{(0)}
$$

and the following hold:

Case (1): (i) $K^{(\eta+i \theta)}=K^{(\eta+i \theta)} \cap(J+\bar{H})=\pi\left(U^{(\eta+i \theta)}\right)$ for all $i \neq 0$; (ii) $K^{(\theta)} \cap M=K^{(\theta)} \cap(J+(\bar{H} \cap M)) \subset \pi\left(U^{(\theta)}\right)$; (iii) $K^{(\theta)} \cap N_{0}=\pi\left(U^{(\theta)}\right)$; and (iv) $K^{(\eta)} \cap\left(J+S_{(0)} \otimes F+\bar{H}\right)=\pi\left(U^{(\eta)}\right)$.

Case (2): (i) $K^{(\eta+i \theta)}=K^{(\eta+i \theta)} \cap\left(J+S_{(0)} \otimes F+\bar{H}\right)=\pi\left(U^{(\eta+i \theta)}\right)$ for all $i$; (ii) $K^{(\eta+i \theta)}=\pi\left(U^{(\eta+i \theta)}\right)$ for all $i \neq \pm 1$; (iii) $K^{(\theta)} \cap M=K^{(\theta)} \cap\left(J+S_{(0)} \otimes F+\right.$ $(\bar{H} \cap M))$; and (iv) $K^{(\theta)} \cap N_{0}=\pi\left(U^{(\theta)}\right)$. Consequently, $\pi\left(U^{(\zeta)}\right)=K^{(\zeta)} \cap N_{0}$ for 
all roots $\zeta \neq 0$. If $L_{\zeta} \not \subset Q \cap L^{(\alpha, \beta)}$, then $\zeta \in\{ \pm \eta, \pm \theta\}$ in case (1). In case (2) there is an $m \neq 0$ such that if $L_{\zeta} \not \subset Q \cap L^{(\alpha, \beta)}$, then $\zeta \in\{ \pm \eta+\theta, m \theta\}$ if $\theta$ is Witt, and $\zeta \in\{ \pm \eta+\theta, \pm m \theta\}$ if $\theta$ is Hamiltonian.

Remark. Just as in Lemma 2.4, in Corollary $2.7(\operatorname{Der} S)_{(0)}=S_{(0)}=S$ when $S=s l(2)$.

Proof. That $K^{(\eta+i \theta)} \subset G+\bar{H}$ follows from the existence of a nonzero toral element $t_{\theta} \in G \cap \operatorname{ker} \theta$. The second paragraph of the proof of Lemma 2.3 shows that $\bar{H} \subset N$. When $S=\operatorname{sl}(2)$ or $W(1: \underline{\underline{1}}), \operatorname{Der} S=\operatorname{ad} S$, and when $S=H(2: \underline{\underline{1}})^{(2)}, \operatorname{Der} S=\operatorname{ad} S+V$ where

$$
V=\left\langle\mathscr{D}\left(x^{p \epsilon_{i}}\right) \otimes 1 \mid i=1,2\right\rangle \oplus\left\langle\left(x^{\epsilon_{1}} D_{1}+x^{\epsilon_{2}} D_{2}\right) \otimes 1\right\rangle \subset(\operatorname{Der} S)_{(0)} .
$$

Therefore $\bar{H} \cap M \subset$ ad $S_{(0)}+V$ (where $V=(0)$ when $S \cong s l(2)$ or $W(1: \underline{1})$ ).

Case (1): Statements (i),(ii), and (iv) follow directly from the proof just completed. For (iii), let $W:=\operatorname{id}_{S} \otimes W(n: \underline{\underline{1}})$; and $W_{(i)}:=\operatorname{id}_{S} \otimes W(n: \underline{\underline{1}})_{(i)}$. Then $N=M \oplus W_{(0)}$. It follows from what we have established in Lemma 2.3 that $K^{(\theta)} \cap M \subseteq J+(\bar{H} \cap G)+V$ Then $K^{(\theta)} \cap M$ is a solvable ideal of $K^{(\theta)} \cap N$. Moreover,

$$
\left(K^{(\theta)} \cap N\right) /\left(K^{(\theta)} \cap M\right) \cong\left(K^{(\theta)} \cap N+M\right) / M \subseteq N / M \cong W_{(0)} .
$$

Since $W_{(0)} / W_{(1)}$ is one-dimensional or isomorphic to $g l(2)$, any subalgebra of $W_{(0)}$ is compositionally classical. Thus, we see that $\left(K^{(\theta)} \cap N\right) /\left(K^{(\theta)} \cap M\right)$ and $K^{(\theta)} \cap N$ are compositionally classical. Lemma 2.3 shows that $\pi\left(U^{(\theta)}\right) \subset N$. Thus, $\pi\left(U^{(\theta)}\right)=K^{(\theta)} \cap N=K^{(\theta)} \cap N_{0}$.

Case (2): The first three statements follow from the proof of Lemma 2.3. For part (iv) observe that $K^{(\theta)} \cap M \subseteq J+S_{(0)} \otimes F+V$ where $V$ is as in case (1). The remainder of the argument is identical to that in case (1).

In either case we have $\pi\left(U^{(\zeta)}\right)=K^{(\zeta)} \cap N_{0}$. In case (1) the only nonclassical 1 -sections are $L^{(\eta)}, L^{(\theta)}$. The assertion is just a matter of normalizing $\eta$ and $\theta$. In case (2) the only nonclassical 1-sections are $L^{(\eta-\theta)}, L^{(\eta+\theta)}, L^{(\theta)}$, and $L^{(\eta-\theta)}, L^{(\eta+\theta)}$ are Witt. Let $\zeta \in \mathbb{Z}_{p}(\eta-\theta)$ be the unique root such that $L_{\zeta} \not \subset$ $U^{(\eta-\theta)}$. Consider the homomorphism $\pi: L^{(\eta-\theta)} \rightarrow \operatorname{Der} S$. Then $\pi\left(L_{\zeta}\right) \not \subset$ $(\operatorname{Der} S)_{(0)}$, and the normalization of Lemma 2.3 yields that $\pi\left(L_{\zeta}\right)$ is spanned by $D_{1}$. Hence $\zeta=-\eta+\theta$. Similarly the root space $L_{\eta+\theta}$ sticks out of $U^{(\eta-\theta)}$. If $\theta$ is Witt, then there is a unique root $\zeta \in \mathbb{Z}_{p} \theta$ with $L_{\zeta} \not \subset U^{(\theta)}$. Let $\zeta=m \theta$. If $\theta$ is Hamiltonian, there are two such roots $m \theta, n \theta$. By Corollary $1.14, n=-m$.

\section{The subalgebra $Q(L, T)$}

In this section we show that $Q=Q(L, T)=\sum_{\alpha \in \Omega(T)} U^{(\alpha)}$ is in fact a subalgebra. The results obtained here are extensions to the general case of results known to hold for restricted simple algebras, (see [BW4, Lemma 12.2.3]).

Theorem 3.1. Let $L$ be a simple Lie algebra over an algebraically closed field $F$ of characteristic $p>7$. Let $T$ be an optimal torus of some p-envelope $\left(L_{p},[p], l\right)$ of $L$ and let $Q=Q(L, T)$ be the subspace defined by (1.12). Then $Q$ is a $T$-invariant subalgebra of $L$. 
Proof. In view of Theorem 1.13 and Corollary 1.14 , it suffices to show that $\left[Q_{\alpha}, Q_{\beta}\right] \subseteq Q_{\alpha+\beta}$ whenever $\alpha, \beta$ are nonzero $\mathbb{Z}_{p}$-independent roots. Hence, we work in the 2-section $L^{(\alpha, \beta)}$ and apply Theorem 1.15. As in that theorem, let $I=I^{(\alpha, \beta)}$, the maximal solvable ideal of $L^{(\alpha, \beta)}+T$, and $K=\pi\left(L^{(\alpha, \beta)}\right)$. Then $I \cap L^{(\alpha)} \subseteq \operatorname{rad} L^{(\alpha)} \subseteq U^{(\alpha)}$. Therefore, we may work in $K$. Choose $x \in Q_{\alpha}$, and $y \in Q_{\beta}$, and let $\bar{x}=\pi(x)$ and $\bar{y}=\pi(y)$.

Consider now the various cases in Theorem 1.15. By the reductions of the previous paragraph, the first case to consider is $(3)$. In this case $[\bar{x}, \bar{y}]=0$, so the result is clear. In cases (4), (7) (with $S=H(2: \underline{1}: \Delta)$ ), and (8) (with $S$ nonclassical), $\bar{x} \in \pi\left(U^{(\alpha)}\right)=K^{(\alpha)} \cap K_{(0)}$, and $\bar{y} \in \pi\left(U^{(\beta)}\right)=K^{(\beta)} \cap K_{(0)}$. Then

$$
[\bar{x}, \bar{y}] \in K_{\alpha+\beta} \cap K_{(0)} \subseteq K^{(\alpha+\beta)} \cap K_{(0)}=\pi\left(U^{(\alpha+\beta)}\right) \subseteq \pi\left(Q \cap L^{(\alpha, \beta)}\right) .
$$

In case (7) (with $\left.S=H(2: \underline{\underline{1}}: \phi(\tau))^{(1)}\right)$ and case (8) (with $S$ classical) $Q \cap$ $L^{(\alpha, \beta)}=L^{(\alpha, \beta)}$, so the result is clear. If $S=W(1: \underline{\underline{2}})$ or $H(2:(2,1))^{(2)}$ in (7), then $\bar{x} \in K^{(\alpha)} \cap S_{(0)}$ and $\bar{y} \in K^{(\beta)} \cap S_{(0)}$ by Lemma 2.5. Thus, $[\bar{x}, \bar{y}] \in$ $K_{\alpha+\beta} \cap S_{(0)} \subset \pi\left(U^{(\alpha+\beta)}\right) \subset \pi\left(Q \cap L^{(\alpha, \beta)}\right)$.

In case (6) we have by Lemma 2.4 that $\pi\left(U^{(\zeta)}\right)=K^{(\zeta)} \cap\left\{(\operatorname{Der} S)_{(0)} \otimes F+\right.$ $\left.(\operatorname{Der} S)^{(1)} \otimes x A(1: \underline{\underline{1}})\right\}$ for all roots $\zeta \neq 0$. Then

$$
[\bar{x}, \bar{y}] \in K_{\alpha+\beta} \cap\left\{(\operatorname{Der} S)_{(0)} \otimes F+(\operatorname{Der} S)^{(1)} \otimes x A(1: \underline{\underline{1}})\right\} \subseteq \pi\left(U^{(\alpha+\beta)}\right) .
$$

Similarly in case (5), for each nonzero root $\zeta$, Corollary 2.7 gives $\pi\left(U^{(\zeta)}\right)=$ $K^{(\zeta)} \cap N_{0}$ where

$$
N_{0}=(\operatorname{Der} S)_{(0)} \otimes F+(\operatorname{Der} S) \otimes\left(\sum_{i} x^{\epsilon_{i}} A(n: \underline{\underline{1}})\right)+\operatorname{id}_{S} \otimes W(n: \underline{\underline{1}})_{(0)} .
$$

Thus since $N_{0}$ is a subalgebra, $[\bar{x}, \bar{y}] \in K_{\alpha+\beta} \cap N_{0}=\pi\left(U^{(\alpha+\beta)}\right)$.

Finally $Q$ is invariant under $T$ since $U^{(\zeta)}$ is $T$-invariant for each root $\zeta$, (compare Theorem 1.13).

Theorem 3.2. Let $L$ be a simple Lie algebra over an algebraically closed field of characteristic $p>7$, and suppose that $T$ is an optimal torus in some p-envelope of $L$. Denote by $Q:=Q(L, T)$ the subalgebra defined by Theorem 3.1, and set $Q^{(\alpha, \beta)}:=Q \cap L^{(\alpha, \beta)}$. Let $J^{(\alpha, \beta)}$ denote the maximal solvable ideal of $Q^{(\alpha, \beta)}+T . S e t$

$$
\begin{gathered}
\varrho:=\varrho(\alpha, \beta): Q^{(\alpha, \beta)}+T \rightarrow\left(Q^{(\alpha, \beta)}+T\right) / J^{(\alpha, \beta)}, \\
M:=M^{(\alpha, \beta)}:=\varrho\left(Q^{(\alpha, \beta)}\right), \\
R:=\varrho(T) .
\end{gathered}
$$

Then $M$ is one of the following.

(A) $M=(0)$. This happens in the following cases of Theorem 1.15 .

(2) with $S=W(1: \underline{1})$,

(3) with $S_{1}=S_{2}=\overline{\bar{W}}(1: \underline{1})$,

(5, case 1) with $\eta, \theta$ Witt,

(6) with $S=W(1: \underline{1})$,

(7) with $S=W(1: \underline{\underline{2}})$. 
(B) $M \cong s l(2)$. This happens in the following cases of Theorem 1.15.

(2) with $S \in\left\{\operatorname{sl}(2), H(2: \underline{1})^{(2)}\right\}$,

(3) with $S_{1}=W(1: \underline{\underline{1}}), S_{2} \in\left\{\operatorname{sl}(2), H(2: \underline{\underline{1}})^{(2)}\right\}$ (or vice versa)

(4)

(5, case 1) with $\eta$ classical or Hamiltonian and $\theta$ Witt, or $\eta$ Witt and $\theta$ Hamiltonian, or $(5$, case 2) with $\theta$ Witt,

(7) with $S=H(2: \underline{\underline{1}}: \Delta)$,

(8) with $S=W(2: \underline{\underline{1}}), K(3: \underline{\underline{1}})$.

(C) $M \cong \operatorname{sl}(2) \oplus \operatorname{sl}(2)$. This happens in the following cases of Theorem 1.15.

(3) with $S_{1}, S_{2} \in\left\{\operatorname{sl}(2), H(2: \underline{1})^{(2)}\right\}$,

(5, case 1$)$ with $\eta$ classical or Hamiltonian and $\theta$ Hamiltonian or (5, case

2) with $\theta$ Hamiltonian.

(D) $M \cong \operatorname{sl}(2) \otimes A(1: \underline{\underline{1}})$. This happens in the following cases of Theorem 1.15 .

(6) with $S \in\left\{\operatorname{sl}(2), H(2: \underline{1})^{(2)}\right\}$

(7) with $S=H(2:(2,1))^{(\overline{2})}$

(E) $M \cong H(2: \underline{\underline{1}}: \phi(\tau))^{(1)}$. This happens in the following case of Theorem 1.15 . (7) with $S=H(2: \underline{\underline{1}}: \phi(\tau))^{(1)}$.

(F) $M \in\left\{A_{2}, C_{2}, G_{2}\right\}$. This happens in the following cases of Theorem 1.15.

(8) with $S=A_{2}, C_{2}, G_{2}, S(3: \underline{\underline{1}})^{(1)}, H(4: \underline{\underline{1}})^{(2)}$.

Proof. The ideal $I^{(\alpha, \beta)}$ of $L^{(\alpha, \beta)}+T$ defined in Theorem 1.15 is contained in $Q^{(\alpha, \beta)}+T$, and in addition it is a solvable ideal. Thus, $I^{(\alpha, \beta)} \subseteq J^{(\alpha, \beta)}$, and $\varrho\left(Q^{(\alpha, \beta)}\right)$ is a homomorphic image of $\pi\left(Q^{(\alpha, \beta)}\right)$ in $K:=K^{(\alpha, \beta)}:=\pi\left(L^{(\alpha, \beta)}\right)$ (in the notation of Theorem 1.15.) Therefore, we have to argue in $K$, and determine the image of $Q^{(\alpha, \beta)}$ in $K$ and its $T$-invariant solvable radical. Then $M$ is obtained as the quotient.

It is easy to see that the assertions are true in cases (1), (2), (3), (7) with $S \cong$ $H(2: \underline{\underline{1}}: \phi(\tau))^{(1)}$, and (8) with $S$ classical.

In cases (4), (7) with $S=H(2: \underline{1}: \Delta)$, and (8) with $S$ nonclassical, $K$ carries a natural filtration (as was pointed out in $\S 2$ ). According to Lemmas $2.2,2.5$, and 2.6 and their proofs, $K_{(i)}$ is invariant under $\bar{T}:=\pi(T)$ for every $i$, and $K_{(0)}$ is the image of $Q^{(\alpha, \beta)}$. Then $M$ is a homomorphic image of $K_{(0)} / K_{(1)}$, which is classical reductive.

In case (5) we apply Corollary 2.7 to obtain that $S \otimes\left(\sum_{i=1}^{n} x^{\epsilon_{i}} A(n: \underline{\underline{1}})\right)$ is a $\pi\left(Q^{(\alpha, \beta)}+T\right)$-invariant ideal which in addition is nilpotent. Thus, there are homomorphisms

$$
\begin{gathered}
\sigma: Q^{(\alpha, \beta)} \rightarrow \pi\left(Q^{(\alpha, \beta)}\right) / \pi\left(Q^{(\alpha, \beta)}\right) \cap S \otimes\left(\sum_{i=1}^{n} x^{\epsilon_{i}} A(n: \underline{\underline{1}})\right), \\
\sigma^{\prime}: \sigma\left(Q^{(\alpha, \beta)}\right) \rightarrow M
\end{gathered}
$$

with solvable $T$-invariant kernels. Lemma 2.3 and Corollary 2.7 yield

$$
\sigma\left(Q^{(\alpha, \beta)}\right)=S_{(0)}+\sigma\left(U^{(\theta)}\right) .
$$

Then $M$ is isomorphic to $S_{(0)} / \operatorname{rad} S_{(0)} \oplus U^{(\theta)} / \operatorname{rad} U^{(\theta)}$. Note that $S_{(0)} / \operatorname{rad} S_{(0)}$ $=(0)$ if we consider case (1) of type (5) and $\eta$ is Witt, and $S_{(0)} / \operatorname{rad} S_{(0)} \cong$ 
$s l(2)$ in all other cases. Moreover, $U^{(\theta)} / \operatorname{rad} U^{(\theta)}=(0)$ for $\theta \mathrm{Witt}$, and $U^{(\theta)} / \operatorname{rad} U^{(\theta)} \cong s l(2)$ for $\theta$ Hamiltonian. This settles case (5).

In case (6) with $S=\operatorname{sl}(2)$ every 1 -section is classical or solvable. Thus, $\pi\left(Q^{(\alpha, \beta)}\right)=K$, and $M$ is of type (D). When $S=W(1: \underline{\underline{1}})$, then $\operatorname{Der} S=\operatorname{ad} S$. Let $\operatorname{Der} S_{(0)} \cong W(1: \underline{\underline{1}})_{(0)}$ denote the maximum compositionally classical subalgebra. We have (compare Lemma 2.4)

$$
\begin{aligned}
\pi\left(Q^{(\alpha, \beta)}\right) & \subseteq(\operatorname{Der} S)_{(0)} \otimes F+(\operatorname{Der} S)^{(1)} \otimes x A(1: \underline{\underline{1}}) \\
& \cong W(1: \underline{\underline{1}})_{(0)} \otimes F+W(1: \underline{\underline{1}}) \otimes x A(1: \underline{\underline{1}}),
\end{aligned}
$$

which is solvable. Thus, $M=(0)$.

In case (7) with $S=W(1: \underline{\underline{2}}), K$ has a solvable subalgebra $W(1: \underline{\underline{2}})_{(0)}$ which, although it is not $\bar{T}$-invariant, contains the image of $Q^{(\alpha, \beta)}$. Thus, $Q^{(\alpha, \beta)}$ is solvable in this case and $M=(0)$.

There are two remaining cases to consider: (6) with $S=H(2: \underline{\underline{1}})^{(2)}$ and (7) with $S=H(2:(2,1))^{(2)}$. In both cases there is a 1 -section of $Q^{(\alpha, \beta)}$ which is solvable and all others are classical. Observe that $Q_{p}^{(\alpha, \beta)}=Q^{(\alpha, \beta)}+\left\langle Q_{\mu}^{[p]^{i}}\right| \mu \in$ $\Omega, i \geq 1\rangle$. Therefore

$$
T \cap\left(Q_{p}^{(\alpha, \beta)}+C\left(T+Q_{p}^{(\alpha, \beta)}\right)\right) \subset H_{p}+C\left(T+Q_{p}^{(\alpha, \beta)}\right) .
$$

Theorem 1.5 of [S4] now implies that $\operatorname{TR}(M) \leq \operatorname{TR}(\bar{H}, K)=1$. Since $M$ is not nilpotent, this means that $\operatorname{TR}(M)=1$. As $M$ is $\bar{T}$-semisimple, there are only two possibilities for $M$ by [S2, Lemma 6.2]:

$$
M \text { is semisimple or } M_{1} \otimes A(1: \underline{\underline{1}}) \subset M \subset \operatorname{Der}\left(M_{1} \otimes A(1: \underline{\underline{1}})\right)
$$

where $M_{1}$ is a simple algebra. When $M$ is semisimple, [S1, Corollary 4.2] applies, and as every 1 -section is solvable or classical, it follows that $M \cong \operatorname{sl}(2)$. Then every 1 -section in $K$ but one is solvable, which is not true in the present case. When $M_{1} \otimes A(1: \underline{\underline{1}}) \subset M \subset \operatorname{Der}\left(M_{1} \otimes A(1: \underline{1})\right)$, the same reasoning gives that $M_{1} \cong s l(2)$, and hence that $s l(2) \otimes A(1: \underline{1}) \subset M \subset \operatorname{sl}(2) \otimes A(1: \underline{\underline{1}}) \oplus F \otimes U$ where $U \subset W(1: \underline{\underline{1}})$. Consider the restricted homomorphism:

$$
\begin{aligned}
\psi: & \operatorname{sl}(2) \otimes A(1: \underline{\underline{1}}) \oplus F \otimes W(1: \underline{\underline{1}}) \\
& \rightarrow(\operatorname{sl}(2) \otimes A(1: \underline{\underline{1}}) \oplus F \otimes W(1: \underline{\underline{1}})) / \operatorname{sl}(2) \otimes A(1: \underline{\underline{1}}) \cong W(1: \underline{\underline{1}}) .
\end{aligned}
$$

Since $s l(2) \otimes x A(1: \underline{\underline{1}})$ is $M$-invariant, $M$ is mapped into $W(1: \underline{\underline{1}})_{(0)}$. But $s l(2) \otimes x A(1: \underline{\underline{1}})$ is not $(M+\bar{T})$-invariant, and therefore $\psi(\bar{T}) \not \subset W(1: \underline{\underline{1}})_{(0)}$. Thus, there is an $\bar{t} \in \bar{T}$ such that $\psi(\bar{t})$ is conjugate under an automorphism $\phi$ of $W(1: \underline{\underline{1}})$ to $(1+x) \partial$. Then $\phi \psi(M) \subset W(1: \underline{\underline{1}})_{(0)}$ is invariant under $(1+x) \partial$, which is possible only if $\phi \psi(M)=0$. Hence $M \subset \operatorname{sl}(2) \otimes A(1: \underline{\underline{1}})$ and $M$ is of type D.

\section{Classification Results}

We recall the notion of a standard filtration determined by a maximal subalgebra $L_{(0)}$ and a subspace $L_{(-1)} \supseteq L_{(0)}$. Let $L_{(0)}$ be a maximal subalgebra of a Lie algebra $L$ not containing a nonzero ideal of $L$. Choose a subspace $L_{(-1)} \supseteq L_{(0)}$ such that $L_{(-1)} / L_{(0)}$ is an irreducible $L_{(0)}$-submodule of $L / L_{(0)}$. Define

$$
L_{(i+1)}:=\left\{x \in L_{(i)} \mid\left[x, L_{(-1)}\right] \subseteq L_{(i)}\right\}, \quad i \geq 0
$$




$$
L_{(i)}:=L_{(i+1)}+\left[L_{(-1)}, L_{(i+1)}\right], \quad i \leq-1 .
$$

The assumptions on $L_{(0)}$ insure that there exist $k, l$ such that $L=L_{(-k)}$ and $L_{(l)}=(0)$. Then $\left[L_{(i)}, L_{(j)}\right] \subseteq L_{(i+j)}$ for all $i, j$, and $L_{(1)}$ acts nilpotently on $L$.

For each subalgebra $G$ of $L$, denote by $R(G, L)$ the maximal ideal of $G$ which acts nilpotently on $L$. In the situation described above, $L_{(1)} \subseteq$ $R\left(L_{(0)}, L\right)$. On the other hand, $R\left(L_{(0)}, L\right)$ acts nilpotently on the irreducible module $L_{(-1)} / L_{(0)}$. The space of vectors annihilated by $R\left(L_{(0)}, L\right)$ is a nonzero $L_{(0)}$-submodule, and so must be $L_{(-1)} / L_{(0)}$. By the definition of $L_{(1)}$, $R\left(L_{(0)}, L\right) \subseteq L_{(1)}$. Thus, $R\left(L_{(0)}, L\right)=L_{(1)}$.

Lemma 4.1. Let $L$ be simple and $G$ be a proper subalgebra. If $[x, L] \subseteq G$ for some $x \in G$, then $x \in R(G, L)$.

Proof. Embed $G$ in a maximal subalgebra $L_{(0)}$, and choose any $L_{(-1)}$ as above. This defines a filtration for which $x \in L_{(1)} \cap G$. The latter is an ideal of $G$ acting nilpotently on $L$, and is therefore contained in $R(G, L)$.

Theorem 4.2. Let $L$ be a simple Lie algebra over an algebraically closed field of characteristic $p>7$. Let $T$ be an optimal torus in some p-envelope and $Q:=Q(L, T)$ the subalgebra constructed in Theorem 3.1. Assume that $Q \neq L$. Let $T^{\prime} \subseteq T$ denote a torus which distinguishes the roots (with respect to $T$ ) on $L / Q$. Then $Q+T^{\prime}$ is a maximal subalgebra of $L+T^{\prime}$.

Proof. Assume that $G$ is a $T^{\prime}$-invariant subalgebra of $L$ such that

$$
Q \varsubsetneqq G \varsubsetneqq L
$$

Since every root space of $L / Q$ with respect to $T$ is one-dimensional and $T^{\prime}$ distinguishes these root spaces, $G$ is not only $T^{\prime}$-invariant, but also $T$ invariant.

(1) As every $T$-module is semisimple, there is a decomposition

$$
G=Q+\sum_{\mu} G \cap L_{\mu}
$$

The construction of $Q$ ensures the existence of a nonclassical root $\alpha$ with

$$
L^{(\alpha)} \cap G \neq L^{(\alpha)} \cap Q .
$$

As $L^{(\alpha)} \cap Q$ is a maximal subalgebra of $L^{(\alpha)}$, this implies that $L^{(\alpha)} \subset G$.

(2) Let $\beta \notin \mathbb{Z}_{p} \alpha$ be a root and consider the 2 -section $L^{(\alpha, \beta)}$, as well as its $T$-semisimple quotient $K:=K^{(\alpha, \beta)}$. Put $G^{\prime}:=\pi\left(G \cap L^{(\alpha, \beta)}\right)$. We now consider the cases in Theorem 1.15. If $K$ is of type (6) or of type (7) with $S=W(1: \underline{\underline{2}})$ or $H(2:(2,1))^{(2)}$, then there is a root $\mu$ with $\mu(\bar{H})=0 \quad(\bar{H}:=\pi(H))$ which has the additional properties [BW4, 10.1.1, 11.1.1; S3, IV.4]

$$
K^{(\mu)} \subseteq \pi\left(G \cap L^{(\alpha, \beta)}\right)=G^{\prime},
$$

there exists $x \in K_{\mu}$ with $\lambda\left(x^{[p]}\right) \neq 0$ for all $\lambda \notin \mathbb{Z}_{p} \mu$.

This element $x$ acts invertibly on $\sum_{i \in \mathbb{Z}_{p}} K_{j \alpha+i \mu}$ for all nonzero $j$. As $L^{(\alpha)}$ is contained in $G$, this implies that $K^{(\alpha)} \subseteq G^{\prime}$, and hence $K \subseteq G^{\prime}$. In all other cases we have

$$
\operatorname{dim} K / G^{\prime} \leq \operatorname{dim} K / \pi\left(Q \cap L^{(\alpha, \beta)}\right) \leq 4 .
$$


Then $K^{(\alpha)}$ acts on a composition series of $K / G^{\prime}$ whose factors have dimension $<p-1$. Since the socle of $K^{(\alpha)}$ is isomorphic to $W(1: \underline{\underline{1}})$ or $H(2: \underline{\underline{1}})^{(2)}$, no homomorphic image of $K^{(\alpha)}$ is classical reductive. This means in light of [Ko] that $\left(K^{(\alpha)}\right)^{(1)}$ acts nilpotently on $K / G^{\prime}$.

(3) Put $N:=\bigcap_{n=0}^{\infty}\left(L^{(\alpha)}\right)^{(n)}$. Then $N \neq(0), N^{(1)}=N$, and according to (2) $\left[N, L^{(\beta)}\right] \subseteq G$ for all nonzero roots $\beta$. Hence $[N, L] \subseteq G$, and Lemma 4.1 implies that $N \subseteq R(G, L)$. Therefore, $N$ would be nilpotent, contradicting the fact that $N^{(1)}=N \neq(0)$. Thus, $G=L$.

(4) As a result of (3) we have that $Q$ is a maximal $T^{\prime}$-invariant subalgebra. Then $Q+T^{\prime}$ is a subalgebra of $L+T^{\prime}$, and since $L \cap T^{\prime} \subseteq H \subseteq Q$, this subalgebra is different from $L+T^{\prime}$. Hence it is maximal.

Theorem 4.3. Let $L$ be a simple Lie algebra over an algebraically closed field of characteristic $p>7$. Suppose $T$ is an optimal torus in some p-envelope of $L$, and let $Q=Q(L, T)$ be the subalgebra constructed in Theorem 3.1. Assume that $Q \neq L$. If $L$ has no 2-section of type (8) with $S \cong K(3: \underline{\underline{1}})$, then there is a subspace $V$ of $L$ with

$$
L=Q \oplus V, \quad[T, V] \subseteq V, \quad V^{(1)} \subseteq Q .
$$

Proof. For any root $\alpha$ we have either $L_{\alpha} \subset Q$ or $\operatorname{dim} L_{\alpha} / L_{\alpha} \cap Q=1$. Let $\Omega$ be the set of roots $\alpha$ with $L_{\alpha} \neq Q_{\alpha}$. For $\alpha \in \Omega$ choose $x_{\alpha} \in L_{\alpha}$ such that $L_{\alpha}=F x_{\alpha}+L_{\alpha} \cap Q$, and define

$$
V:=\sum_{\alpha \in \Omega} F x_{\alpha}
$$

We have to prove that $\left[x_{\alpha}, x_{\beta}\right] \in Q$ for all $\alpha, \beta \in \Omega$.

If $\alpha, \beta$ are $\mathbb{Z}_{p}$-dependent, then $\alpha$ is Hamiltonian and $\beta=-\alpha$. In this case $\left[x_{\alpha}, x_{\beta}\right] \in H \subset Q$.

Let $\alpha, \beta$ be $\mathbb{Z}_{p}$-independent roots in $\Omega$. Then $L^{(\alpha, \beta)}$ cannot be of types (1), (2), (7) with $S=H(2: \underline{\underline{1}}: \phi(\tau))^{(1)}$, or $(8)$ with $S$ classical of Theorem 1.15, since in these cases there is at most one nonclassical, nonsolvable 1-section. In cases (3), (4), (5, case 1), (7) with $S=H(2: \underline{\underline{1}}: \Delta)$, or (8) with $S=W(2: \underline{\underline{1}})$ or $H\left(4: \underline{1}^{(2)}\right.$, there are at most two nonsolvable and nonclassical 1-sections, which then have to be $L^{(\alpha)}, L^{(\beta)}$. Hence, $\left[x_{\alpha}, x_{\beta}\right] \in L_{\alpha+\beta} \subset Q$. For $(5$, case 2$)$ the only possible roots sticking out of $Q$ are $\pm \eta+\theta, \pm m \theta$. If $\alpha, \beta, \alpha+\beta$, are of that form, then $\alpha=\eta+\theta$ and $\beta=-\eta+\theta$. Then $K_{\alpha}, K_{\beta}$ are contained in $S \otimes A(n: \underline{\underline{1}})$, and hence $\left[x_{\alpha}, x_{\beta}\right] \in \operatorname{rad} L^{(\theta)} \subset Q$.

In case (6) or case (7) with $S=W(1: \underline{\underline{2}})$ or $S=H(2:(2,1))^{(2)}$, there are roots $\eta, \theta$ with $\eta(H) \neq 0, \theta(H)=0$ such that $\eta \in \Omega$, and the only roots in $\Omega$ are $\eta+i \theta$ for all $i \in \mathbb{Z}_{p}$ (when $\eta$ is Witt) and $\pm \eta+i \theta$ for all $i \in \mathbb{Z}_{p}$ (when $\eta$ is Hamiltonian). Thus, if $\alpha, \beta$ are of this form, then $\alpha+\beta$ is not, and $L_{\alpha+\beta} \subset Q$.

Finally, in case (8) with $S=S(3: \underline{\underline{1}})^{(1)}$, there are exactly three roots sticking out of $Q$. None of them is the sum of the other two, so the result is clear in this case. Since case (8) with $S=K(3: \underline{\underline{1}})$ is assumed not to occur, this completes the proof.

Corollary 4.4. With assumptions as in Theorem 4.3, let $T^{\prime} \subseteq T$ denote a torus which distinguishes roots on $L / Q$. Then $L / Q$ is an irreducible $Q+T^{\prime}$-module. 
Proof. Assume that $W$ is a $Q+T^{\prime}$-submodule of $L$ with $Q \varsubsetneqq W$. Choose $V$ as in Theorem 4.3. Then $W=Q+V \cap W$. Suppose that $x, y \in W$. If $x \in Q$ or $y \in Q$, then $[x, y] \in W$. If both lie outside $Q$, then Theorem 4.3 yields $[x, y] \in Q$. Thus, $W$ is a $T^{\prime}$-invariant subalgebra properly containing $Q$. The result follows from Theorem 4.2 .

In the situation of Theorem 4.2, $Q+T$ is a maximal subalgebra of $L+T$, which as $L$ is simple, contains no proper ideals of $L+T$. Thus, choosing a suitable $(Q+T)$-invariant subspace $L_{(-1)}$ of $L$ containing $Q$ such that $L_{(-1)} / Q$ is $(Q+T)$-irreducible, we set $(L+T)_{(-1)}:=L_{(-1)}+T$. This defines a standard filtration of $L+T$. In this context, we will use a slightly different notation than that introduced at the beginning of the section, as we are interested in $T$-invariant filtrations of $L$. Put

$$
Q_{(i)}:=L_{(i)} \text { for } i>0 .
$$

Then

$$
L+T \supset \cdots \supset(L+T)_{(0)}=Q+T \supset L_{(1)}=Q_{(1)} \supset \cdots
$$

defines a standard filtration of $L+T$ and a $T$-invariant filtration of $L$

$$
L \supset \cdots \supset L_{(-1)} \supset Q_{(0)}:=Q \supset Q_{(1)} \supset \cdots
$$

with $Q_{(i+1)}=\left\{x \in Q_{(i)} \mid\left[x, L_{(-1)}\right] \subset Q_{(i)}\right\},(i \geq 0)$.

Theorem 4.5. Let $L$ be a simple Lie algebra over an algebraically closed field of characteristic $p>7$. Assume $T$ is an optimal torus in some p-envelope and let $Q$ be the subalgebra constructed in Theorem 3.1. Assume that $Q \neq L$, and no 2-section of type (8) with $S=K(3: \underline{1})$ occurs. Let $\alpha$ denote a nonsolvable root. Then

$$
\operatorname{rad}\left(Q \cap L^{(\alpha)}\right) \subset H+Q_{(1)}
$$

Proof. Let $\beta$ denote any nonclassical root, and consider the $T$-semisimple 2-section $K:=K^{(\alpha, \beta)}=\pi\left(L^{(\alpha, \beta)}\right)$. Take any $x \in \operatorname{rad}\left(L^{(\alpha)} \cap Q\right)$ and put $y:=\pi(x)$.

If $L_{\beta} \subset Q$ or $L_{\alpha+\beta} \subset Q$, then $\left[x, L_{\beta}\right] \subset Q$. Thus, we consider the case that $L_{\beta}, L_{\alpha+\beta} \not \subset Q$ and that $\alpha$ is nonsolvable. Then $L^{(\alpha, \beta)}$ is not of types (1), (2), (3), (5, case 1), (6), (7) with $S=W(1: \underline{2}), H(2:(2,1))^{(2)}$, or $H(2: \underline{1}: \phi(\tau))^{(1)}$, or $(8)$ with $S$ classical. When $L^{(\alpha, \beta)}$ is of type (5, case 2$)$, the only possibility is that $\alpha= \pm 2 \eta$ and $\beta= \pm \eta+\theta$. Then $K_{\alpha}, K_{\beta} \subset S \otimes A(1: \underline{\underline{1}})$ and

$$
\operatorname{rad}\left(K^{(\alpha)} \cap \pi(Q)\right) \subset(\operatorname{Der} S)_{(1)} \otimes A(n: \underline{\underline{1}})+S \otimes \sum x_{i} A(n: \underline{\underline{1}}) .
$$

Therefore, $\left[x, L_{\beta}\right] \subset Q$. To discuss the remaining cases - (4), (7) with $S=$ $H(2: \underline{1}: \Delta)$, and (8) with $S$ nonclassical but not $K(3: \underline{\underline{1}})$, we observe that $x$ was chosen in the radical of $L^{(\alpha)} \cap Q$. In all these cases $y \in \bar{H}+(\operatorname{Der} S)_{(1)}$ is true, and therefore $\left[y, K_{\beta}\right] \subset \pi\left(Q \cap L^{(\alpha, \beta)}\right)$. As a result, $\left[x, L_{\beta}\right] \subset Q$ for all nonclassical roots. Consequently, $[x, L] \subset Q$, showing that $x \in Q_{(1)}$, as desired.

This theorem enables us to compare the natural filtration on nonclassical 1sections with the filtration imposed on these 1-sections by the filtration of $L$ itself. The filtration on the 1-section is obtained as follows. Suppose that $\alpha$ is a Witt or Hamiltonian root, $\sigma: L^{(\alpha)} \rightarrow L^{(\alpha)} / \operatorname{rad} L^{(\alpha)}$ is the natural projection, 
and $U^{(\alpha)}$ the maximal $T$-invariant compositionally classical subalgebra. Then $\sigma\left(L^{(\alpha)}\right)$ carries a natural filtration defined by $\sigma\left(U^{(\alpha)}\right)$. Let $L_{(i)}^{(\alpha)}$ be the inverse image of the $i$ th part of that filtration, so in particular, $L_{(0)}^{(\alpha)}=U^{(\alpha)}$.

Corollary 4.6. (1) If $\alpha$ is Witt, then

$$
\begin{gathered}
L_{(i)}^{(\alpha)} \subset Q_{(i-1)}+\operatorname{rad} L^{(\alpha)} \quad(i \geq 1), \\
L_{(1)}^{(\alpha)} \subset Q_{(1)}+H .
\end{gathered}
$$

(2) If $\alpha$ is Hamiltonian, then

$$
L_{(i)}^{(\alpha)} \subset Q_{(i)}+H+\operatorname{rad} L^{(\alpha)} .
$$

(3) $Q_{(p-3)} \neq(0)$.

Proof. (1) If $\alpha$ is Witt, then $U^{(\alpha)}$ is solvable. According to Theorem 4.5, $U^{(\alpha)} \subset H+Q_{(1)}$. This proves the second statement. Considering $\sigma: L^{(\alpha)} \rightarrow$ $L^{(\alpha)} / \operatorname{rad} L^{(\alpha)} \cong W(1: \underline{\underline{1}})$, we observe that we can find elements $e_{1}, e_{2} \in Q_{(1)}$ such that $L_{(i)}^{(\alpha)}=F\left(\operatorname{ad} e_{1}\right)^{i-2} e_{2}+L_{(i+1)}^{(\alpha)}+\operatorname{ker} \sigma$ for every $i \in\{2, \ldots, p-2\}$ (just choose $e_{1}, e_{2}$ so that $\sigma\left(e_{1}\right)=x^{2} d / d x$ and $\left.\sigma\left(e_{2}\right)=x^{3} d / d x\right)$. Therefore $L_{(i)}^{(\alpha)} \subset Q_{(i-1)}+\operatorname{rad} L^{(\alpha)}$, as asserted.

(2) If $\alpha$ is Hamiltonian, then $U^{(\alpha)} / \operatorname{rad} U^{(\alpha)}$ is isomorphic to $\operatorname{sl}(2)$. It is well-known that $H(2: \underline{\underline{1}})_{(i)}^{(2)}$ is the span of $i$-fold products of elements of $H(2: \underline{1})_{(1)}^{(2)}$. (Compare [SF, 4.7.2].) Lifting this information to $L^{(\alpha)}$, we obtain in this case

$$
\begin{aligned}
L_{(i)}^{(\alpha)} & \subset\left\{L_{(1)}^{(\alpha)}\right\}^{i}+H+\operatorname{rad} L^{(\alpha)} \subset Q_{(1)}^{i}+H+\operatorname{rad} L^{(\alpha)} \\
& \subset Q_{(i)}+H+\operatorname{rad} L^{(\alpha)} .
\end{aligned}
$$

(3) In bo'll cases we have $(0) \neq L_{(p-2)}^{(\alpha)} \subset Q_{(p-3)}$.

A further direct consequence of Theorem 4.3 is the following classification result.

Theorem 4.7. Let $L$ be a simple Lie algebra over an algebraically closed field of characteristic $p>7$. Assume $T$ is an optimal torus in some p-envelope and let $Q=Q(L, T)$ be the subalgebra constructed in Theorem 3.1. If $Q$ is solvable, then $L$ is isomorphic to $W(1: \underline{\underline{n}})$ for some $n \geq 1$.

Proof. We first observe that $Q \neq L$ (as $Q$ is solvable and $L$ is simple), and $U^{(\alpha)}$ is solvable for all roots $\alpha$. In particular, no 2-section of $L$ is of type $K(3: \underline{1})$. By Theorem 4.3 , we can choose a subspace $V$ with $L=Q+$ $V, V^{(\overline{1})} \subset Q$. Let $L_{(0)}$ be a maximal $Q$-invariant subspace of $L$ containing $Q$. Then $L_{(0)}=Q+L_{(0)} \cap V$ is a maximal subalgebra of $L$, and $L / L_{(0)}$ is an irreducible $L_{(0)}$-module. Consider the associated standard filtration

$$
L=L_{(-1)} \supset L_{(0)} \supset L_{(1)} \supset \cdots .
$$

Then $\left[L, L_{(0)} \cap V\right] \subset V^{(1)}+\left[Q, L_{(0)} \cap V\right] \subset L_{(0)}$. Hence $L_{(0)} \cap V \subset L_{(1)}$ and $L_{(0)} / L_{(1)} \cong Q / Q \cap L_{(1)}$. The solvability of $Q$ and $L_{(1)}$ implies that $L_{(0)}$ is also solvable. As $L_{0}$ is a maximal subalgebra, $[\mathrm{Ku}]$ applies and yields the result. 


\section{REFERENCES}

[B1] G. Benkart, Cartan subalgebras in Lie algebras of Cartan type, Lie Algebras and Related Topics, Proc. Summer Seminar, 1984, (D. Britten, F. Lemire, and R. Moody, eds.), Canad. Math. Soc. Conf. Proc., vol. 5, Amer. Math. Soc., Providence, RI, 1986, pp. 157-187.

[B2] _ Simple modular Lie algebras with 1-sections that are classical or solvable, Comm. Algebra 18 (1990), 3633-3638.

[B3] _ Simple modular Lie algebras with a 1-section that is Witt or Hamiltonian, preprint.

[BG] G. Benkart and T. Gregory, Graded Lie algebras with classical reductive null component, Math. Ann. 285 (1989), 85-98.

[BGP] G. Benkart, T. Gregory, and A. Premet, The recognition theorem for graded Lie algebras of prime characteristic (in preparation).

[BO] G. M. Benkart and J. M. Osborn, Rank one Lie algebras, Ann. of Math. (2) 119 (1984), 437-465.

[BW1] R. E. Block and R. L. Wilson, The simple Lie p-algebras of rank two, Ann. of Math. 115 (1982), 93-186.

[BW2] _ The restricted simple Lie algebras are of classical or Cartan type, Proc. Nat. Acad. Sci. U.S.A. 81 (1984), 5271-5274.

[BW3] __, Restricted simple Lie algebras, Lie Algebras and Related Topics, Proc. Summer Seminar, (D. Britten, F. Lemire, and R. Moody, eds.), Canad. Math. Soc. Conf. Proc., vol. 5, Amer. Math. Soc., Providence, RI, 1986, pp. 3-17.

[BW4] __, Classification of the restricted simple Lie algebras, J. Algebra 114 (1988), 115-259.

[D1] S. P. Demuškin, Cartan subalgebras of the simple Lie p-algebras $W_{n}$ and $S_{n}$, Sibirsk. Mat. Zh. 11 (1970), 310-325; English transl. in Siberian Math. J. 11 (1970), 233-245.

[D2] _ Cartan subalgebras of simple nonclassical Lie p-algebras, Izv. Akad. Nauk SSSR Ser. Mat. 36 (1972), 915-932; English transl. in Math USSR-Izv. 6 (1972), 905-924.

[Kac1] V. G. Kac, Global Cartan pseudogroups and simple Lie algebras of characteristic $p$, Uspekhi Mat. Nauk 26 (1971), 199-200.

[Kac2] _ - The classification of the simple Lie algebras over a field with nonzero characteristic, Izv. Akad. Nauk SSSR Ser. Mat. 34 (1970), 385-408; English transl. in Math. USSR-Izv.4 (1970), 391-413.

[Kac3] _ Description of filtered Lie algebras with which graded Lie algebras of Cartan type are associated, Izv. Akad. Nauk SSSR Ser. Mat. 38 (1974), 800-834, Errata 40 (1976), 1415; English transl. in Math. USSR-Izv. 8 (1974), 801-835, Errata 10 (1976), 1339.

[Kap] I. Kaplansky, Lie algebras of characteristic p , Trans. Amer. Math. Soc. 89 (1958), 149-183.

[Ko] A. I. Kostrikin, A theorem on semisimple Lie p-algebras, Mat. Zametki 2 (1967), 465-474; English transl. in Math. Notes 2 (1967), 773-778.

[KS1] A. I. Kostrikin and I. R. Šafarevič, Cartan pseudogroups and Lie p-algebras, Dokl. Akad. Nauk SSSR 168 (1966), 740-742; Soviet Math. Dokl. 7 (1966), 715-718.

[KS2] __ Graded Lie algebras of finite characteristic, Izv. Akad. Nauk SSSR Ser. Mat. 33 (1969), 251-322; English transl. in Math. USSR-Izv. 3 (1969), 237-304.

[Ku] M. I. Kuznetsov, Simple modular Lie algebras with a solvable maximal subalgebra, Math. USSR-Sbor. 30 (1976), 68-76.

[MS] W. H. Mills and G. B. Seligman, Lie algebras of classical type, J. Math. Mech. 6 (1957), 519-548.

[SF] H. Strade and R. Farnsteiner, Modular Lie algebras and their representations, Pure and Appl. Math., vol. 116, Dekker, New York, 1988.

[S1] H. Strade, The absolute toral rank of a Lie algebra, Workshop on Lie Algebras (G. Benkart and J. M. Osborn, eds.) Lecture Notes in Math., vol. 1373. Springer-Verlag, Berlin and New York (1989), 1-28.

[S2] _ The classification of simple modular Lie algebras. I: Determination of the two-sections, Ann. of Math. (2) 130 (1989), 643-677. 
[S3] _ The classification of the simple modular Lie algebras. II: The toral structure, J. Algebra (to appear).

[S4] _ The classification of the simple modular Lie algebras. III: Solution of the classical case, Ann. of Math. (2) 133 (1991), 577-604.

[S5] _ New methods for the classification of the simple modular Lie algebras, Mat. Sb. 181 (1990), 1391-1402.

[S6] _ Representations of the $\left(p^{2}-1\right)$-dimensional algebras of $R$. E. Block, Canad. J. Math (to appear).

[S7] _ The classification of the simple modular Lie algebras. IV: The determination of the associated graded algebra, Ann. of Math. (to appear).

[S8] _ The classification of the simple modular Lie algebras. V: Algebras with a nonsolvable 1-section, Abh. Math. Sem. Univ. Hamburg (to appear).

[SW1] H. Strade and R. L. Wilson, Classification of simple Lie algebras over algebraically closed fields of prime cilaracteristic, Bull. Amer. Math. Soc. 24 (1991), 357-362.

[SW2] __, The classification of the simple modular Lie algebras. VI: Solution of the final case (in preparation).

[W1] R. L. Wilson, A structural characterization of the simple Lie algebras of generalized Cartan type over fields of prime characteristic, J. Algebra 40 (1976), 418-465.

[W2] _ Simple Lie algebras of toral rank one, Trans. Amer. Math. Soc. 236 (1978), 287-295.

[W3] __ Classification of the restricted simple Lie algebras with toral Cartan subalgebras, J. Algebra 83 (1983), 531-570.

[W4] _- Simple Lie algebras over fields of prime characteristic, Proc. Internat. Congress Math. (Berkeley, 1986), Vol. I, Amer. Math. Soc., Providence, RI, 1987, pp. 407-416.

Department of Mathematics, University of Wisconsin, Madison, Wisconsin 53706-1388

E-mail address: benkart@math.wisc.edu

E-mail address: osborn@math.wisc.edu

Mathematisches Seminar der Universität Hamburg, Bundesstrasse 55, 2000 Hamburg 13 GERMANY

E-mail address: strade@geomat .math.uni-hamburg.de 